\title{
TET enzymes augment AID expression via 5hmC modifications at the Aicda superenhancer
}

Chan-Wang J. Lio ${ }^{1,8}$, Vipul Shukla ${ }^{1,8}$, Daniela Samaniego-Castruita ${ }^{1,9}$, Edahi González-Avalos ${ }^{1,9}$, Abhijit Chakraborty ${ }^{2}$, Xiaojing Yue ${ }^{1}$, David G. Schatz ${ }^{6,7}$, Ferhat $\mathrm{Ay}^{2}$, Anjana Rao ${ }^{1,3,4,5}$

${ }^{1}$ Division of Signaling and Gene Expression, La Jolla Institute, San Diego, CA; United States;

${ }^{2}$ Division of Vaccine Discovery, La Jolla Institute, San Diego, CA; United States;

${ }^{3}$ Sanford Consortium for Regenerative Medicine, San Diego, CA, United States;

${ }^{4}$ Department of Pharmacology, University of California, San Diego, San Diego, CA, United States;

${ }^{5}$ Moores Cancer Center, University of California, San Diego, San Diego, CA, United States;

${ }^{6}$ Department of Immunobiology, Yale University School of Medicine, New Haven, CT, United States;

${ }^{7}$ Howard Hughes Medical Institute, New Haven, CT, United States.

${ }^{8}$ Equal contribution

${ }^{9}$ Equal contribution

Correspondence should be addressed to A.R. (arao@lji.org)

\section{Abstract}

TET enzymes are dioxygenases that promote DNA demethylation by oxidizing the methyl group of 5-methylcytosine $(5 \mathrm{mC})$ to 5 -hydroxymethylcytosine $(5 \mathrm{hmC})$. Here we report a close correspondence between $5 \mathrm{hmC}$-marked regions, chromatin accessibility and enhancer activity in B cells, and a strong enrichment for consensus binding motifs for basic region-leucine zipper (bZIP) transcription factors at TET-responsive genomic regions. Functionally, Tet2 and Tet3 regulate class switch recombination (CSR) in murine B cells by enhancing expression of Aicda, encoding the cytidine deaminase AID essential for CSR. TET enzymes deposit $5 \mathrm{hmC}$, demethylate and maintain chromatin accessibility at two TET-responsive elements, TetE1 and TetE2, located within a superenhancer in the Aicda locus. Transcriptional profiling identified BATF as the bZIP transcription factor involved in TET-dependent Aicda expression. $5 \mathrm{hmC}$ is not deposited at TetE1 in activated Batf-deficient B cells, indicating that BATF recruits TET proteins to the Aicda enhancer. Our data emphasize the importance of TET enzymes for bolstering AID expression, and highlight $5 \mathrm{hmC}$ as an epigenetic mark that captures enhancer dynamics during cell activation.

\section{Keywords}

Ten-eleven translocation, TET, 5hmC, Aicda, AID, BATF, DNA demethylation, class switch recombination, CSR, epigenetics 


\section{Introduction}

TET proteins (Ten-Eleven-Translocation; TET1, TET2, TET3) are Fe(II)- and a-ketoglutarate-dependent dioxygenases that catalyze the step-wise oxidation of 5-methylcytosine $(5 \mathrm{mC})$ to 5 -hydroxymethylcytosine $(5 \mathrm{hmC}), 5$-formylcytosine $(5 \mathrm{fC})$ and 5-carboxylcytosine $(5 \mathrm{caC})(1,2)$. Together these oxidized methylcytosine (oxi-mC) bases are intermediates in DNA demethylation, and may also function as stable epigenetic marks. $5 \mathrm{hmC}$, the most stable and abundant product of TET enzymatic activity, is highly enriched at the most active enhancers and in the gene bodies of the most highly expressed genes, and its presence at enhancers correlates with chromatin accessibility. TET proteins regulate several fundamental biological processes including lineage commitment, and play important roles in embryonic, neuronal and haematopoietic development (3).

TET proteins, particularly TET2 and TET3, have critical roles in B cell differentiation and malignancy (2). We and others have previously shown that deletion of the Tet2 and Tet3 with Mb1-Cre at early stages of mouse B cell development resulted in impaired light chain rearrangement and developmental blockade, and eventually developed an acute precursor-B-cell-derived leukemia with $100 \%$ penetrance $(4,5)$. Inducible deletion of Tet1 and Tet2 using Mx1-Cre promoted the development of acute lymphoblastic leukemia derived from precursor $\mathrm{B}$ cells, and global loss of Tet1 caused B cell lymphomas with an extended latency (6). In humans, TET2 mutations are frequently observed in Diffuse Large B Cell Lymphoma (DLBCL), a malignancy derived from germinal center B cells $(7,8)$, suggesting that TET proteins may regulate mature B cell function. However, due to the pleiotropic functions of TET proteins, studies of TET-mediated gene regulation are best performed in systems where TET genes are deleted acutely rather than during development.

After their development in the bone marrow, mature B cells migrate to peripheral lymphoid tissues where they encounter antigen and follicular $T$ helper cells in germinal centers, and participate in the generation of functional immune responses(9). In germinal centers, B cells undergo Class Switch Recombination (CSR) to replace the constant region of immunoglobulin $M(\lg M)$ to other isotypes such as $\lg _{1}$ and $\lg A$ for distinct effector functions, and also diversify the variable regions of Ig heavy and light chains for antigen recognition in a process known as somatic hypermutation (SHM). CSR and SHM are both orchestrated by the enzyme AID (Activation-induced cytidine deaminase, encoded by Aicda) (10-12). AID promotes CSR and SHM by generating DNA double-strand breaks at $\mathrm{lg}$ switch regions and point mutations at $\lg$ variable regions, respectively (13). Due to its high mutagenic potential $(14,15)$, AID expression is normally restricted to activated B cells, stimulated either through their B cell receptor and CD40, or through pattern recognition receptors such as TLR4 which binds lipopolysaccharide (LPS).

At the transcriptional level, induction of Aicda is tightly controlled by at least 6 conserved cis-regulatory elements (16). A promoter-proximal region (Region I) and an intronic regulatory element (Region II or E4) function as an enhancer in activated $B$ cells and, in the case of Region II/E4, as a silencer in non-B cells and naïve and memory $B$ cells $(17,18)$. Two additional enhancers, located at $-8 \mathrm{~kb}$ and $+13 \mathrm{~kb}$ relative to the Aicda transcription start site (TSS), are essential for Aicda expression in activated B cells (19). The $+13 \mathrm{~kb}$ region (termed CNS-X, E5, or Region III) showed a dramatic increase in histone H3 acetylation after TLR4-dependent activation. Both the $-8 \mathrm{~kb}$ and $+13 \mathrm{~kb}$ enhancers are necessary for regulating Aicda expression, as shown by studies using bacterial artificial chromosome (BAC) transgenic mice $(17,19)$. Moreover, elements at $-26 \mathrm{~kb}$ (E1) and $-21 \mathrm{~kb}(\mathrm{E} 2)$ are necessary for CSR in CH12F3 B cell line (20), suggesting that the concerted action of all enhancers are required for Aicda expression. The Aicda regulatory elements are occupied by multiple transcription factors, including NFKB, PAX5, E2A, and others $(18,21)$. Transcriptional activation of the Aicda gene is also coupled to cell proliferation, although the mechanistic link between these events is not fully understood (22).

Here we have investigated the role of TET proteins at a kinetic level during B cell activation, integrating our analyses of TET proteins and $5 \mathrm{hmC}$ deposition with data from previous studies that have dissected the transcriptional and epigenetic changes occurring in activated B cells $(20,23)$. We used the Cre ${ }^{E R T 2}$ system for 
acute gene deletion to avoid secondary effects caused by prolonged TET deficiency during cell differentiation. We show that the TET proteins Tet2 and Tet 3 are important regulators of CSR in activated murine B cells and that they function by controlling the activation-induced upregulation of AID mRNA and protein. We further demonstrate that transcriptionally, TET proteins act downstream of the bZIP transcription factor Batf, which is also induced during $B$ cell activation with more rapid kinetics than Aicda, and binds concomitantly with TET proteins to two TET-responsive elements in the Aicda locus that we termed TetE1 and TetE2. Together, our study constitutes a comprehensive analysis of the role of TET proteins in class switch recombination in activated B cells, and provides a detailed analysis of how TET proteins influence cell activation and differentiation.

\section{Results}

\section{Genome-wide kinetics of $5 \mathrm{hmC}$ deposition during B cell activation}

We used CMS-IP $(24,25)$ to analyze the kinetics of genome-wide $5 \mathrm{hmC}$ distribution in murine B cells activated with LPS and IL-4, a well-characterized in vitro system for studying gene regulation (Fig. 1a). The vast majority of $5 \mathrm{hmC}$-marked regions $(\sim 160,000)$ were shared between pre- and post-activated B cells (Fig. 1b, Fig. S1a); of the $~ 9,500$ differentially hydroxymethylated regions (DhmRs) in $72 \mathrm{~h}$-activated versus naïve $B$ cells, the majority $(8,454)$ showed increased $5 \mathrm{hmC}\left(\mathrm{DhmR}^{72 \mathrm{~h} \text {-up }}\right)$ whereas a much smaller fraction showed a decrease $\left(D^{2} R^{\text {down }}\right)$ (Fig. 1b,c). DhmRs were typically located more than $10 \mathrm{~kb}$ from the closest transcription

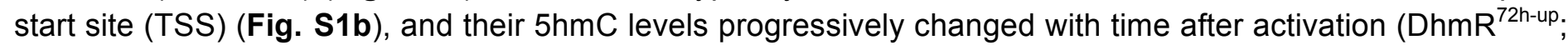
Fig. 1d; DhmR ${ }^{72 h-d o w n} ;$ Fig. S1c).

The oxidized methylcytosines produced by TET proteins are known intermediates in DNA demethylation (2, 26). To relate changes in $5 \mathrm{hmC}$ to changes in DNA methylation, we compared $5 \mathrm{hmC}$ distribution in naïve and 72h-activated B cells with published whole-genome bisulfite sequencing (WGBS) data on B cells activated for $48 \mathrm{~h}$ under similar conditions (20). Although WGBS cannot distinguish $5 \mathrm{mC}$ and $5 \mathrm{hmC}(27), 5 \mathrm{hmC}$ is typically a small fraction (1-10\%) of $5 \mathrm{mC}(28)$, thus we refer to the WGBS signal as "DNA methylation" here. As expected,

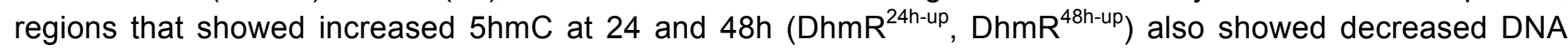
methylation at their centers at $48 \mathrm{~h}$ (Fig. 1e). In contrast, only about half (541/1097) of the differentially methylated regions (DMRs) with decreased DNA methylation at $48 \mathrm{~h}$ (DMR ${ }^{48 \mathrm{~h}-\text { down }}$ ) had increased $5 \mathrm{hmC}$ $\left(\mathrm{DhmR}^{72 \mathrm{~h}-\text { up }}\right.$, Fig. S1d-f), indicating that changes in $5 \mathrm{hmC}$ are a more sensitive measure of epigenetic changes in DNA cytosine modification than changes in $5 \mathrm{mC}$.

Motif enrichment analysis of sequences contained in the $8,454 \mathrm{DhmR}^{72 \mathrm{~h} \text {-up }}$ peaks (Fig. 1c) and the independent set of 1,097 DMR ${ }^{48 h-d o w n}$ regions (Fig. S1d) showed that both sets of regions were enriched in consensus binding sequences for transcription factors of the NFKB (Rel homology domain, RHD) and basic region-leucine (bZIP) families, as well as for "composite" IRF:bZIP motifs (Fig. 1f, Fig. S1g) (29-31). These motifs were not significantly enriched in the $\mathrm{DhmR}^{72 \mathrm{~h} \text {-down }}$ regions ( $<1.5$-fold over background; not shown), pointing to an association between bZIP and NFKB transcription factors and TET-mediated 5hmC deposition.

To discern the relationship between $5 \mathrm{hmC}$ and enhancers, naïve and activated $\mathrm{B}$ cell enhancers, defined by H3K4 monomethylation (H3K4me1), were stratified based on the level of H3K27 acetylation (H3K27Ac), a modification that tracks with enhancer activity (32). In both sets of enhancers, $5 \mathrm{hmC}$ was most highly enriched at active $\left(\mathrm{H} 3 \mathrm{~K} 4 \mathrm{me}^{+} \mathrm{H} 3 \mathrm{~K} 27 \mathrm{Ac}^{+}\right)$relative to poised $\left(\mathrm{H} 3 \mathrm{~K} 4 \mathrm{me} 1^{+} \mathrm{H} 3 \mathrm{~K} 27 \mathrm{Ac}{ }^{-}\right)$enhancers (Fig. 1g). Moreover, more than $75 \%$ of previously identified superenhancers in activated B cells, defined by H3K27Ac, overlapped with at least one $\mathrm{DhmR}^{72 \mathrm{~h} \text {-up }}$ region (Fig. 1h) (33). As an example, a 3' distal element at the Ccr4 locus showed activation-dependent gain of $5 \mathrm{hmC}$ and $\mathrm{H} 3 \mathrm{~K} 27 \mathrm{Ac}$, associated with concomitant loss of methylation at specific CpGs and increased mRNA expression (Fig.s 1i, j). 5hmC was also associated with accessible chromatin defined by ATAC-seq $(4,5,34)$ (see below), and kinetic analysis of active enhancers, defined as differentially active between naïve and $48 \mathrm{~h}$-activated $B$ cells by high accessibility and high H3K27Ac, showed that $5 \mathrm{hmC}$ 
level positively correlated with enhancer activity (Fig. S1h). Together our data show that 5hmC modification and DNA demethylation correlates with enhancer activity during B cell activation.

\section{Comparison of WT and Tet2/3 DKO B cells identifies TET-responsive regulatory elements}

Tet2 and Tet3 are the two major TET proteins expressed in B cells (Fig. 2a). To evaluate the role of TET proteins in regulating $\mathrm{B}$ cell function, we generated mice conditionally deficient in Tet2 and Tet3, using Cre ${ }^{E R T 2}$ and further introduced a Rosa26-LSL-YFP cassette to monitor Cre recombinase activity after tamoxifen treatment (LSL: LoxP-STOP-LOXP cassette in which a strong transcriptional stop is flanked by LoxP sites). Cre ${ }^{E R T 2}$ Tet2 $^{f / f l}$ Tet3 $^{f / f l l}$ Rosa26-LSL-YFP (DKO) and control Tet2 ${ }^{\text {fl/fl }}$ Tet3 ${ }^{f / f l}$ Rosa26-LSL-YFP (WT) mice were treated for 5 days with tamoxifen, after which WT and Tet2/3 DKO B cells were isolated and activated with LPS and IL-4 (Fig. 2b). Both Tet2 and Tet3 were efficiently deleted in B cells (Fig. 2c), and the YFP ${ }^{+}$cells showed similar frequencies of mature splenic follicular B cells (Fig. S2a).

Global $5 \mathrm{hmC}$ levels assessed by DNA dot blot were similar in WT and Tet2/3 DKO B cells prior to activation but showed a perceptible decrease by $48 \mathrm{~h}$ after activation, as expected since $5 \mathrm{hmC}$ is passively lost as a function of cell division $(2,26)$ (Fig. S2b). Starting at $48 \mathrm{~h}, 5 \mathrm{hmC}$ levels were significantly lower in Tet2/3 DKO compared to WT B cells, indicating that Tet2 and Tet3 actively oxidize $5 \mathrm{mC}$ to $5 \mathrm{hmC}$ during B cell activation (Fig. S2b). Around 2,300 5hmC-enriched regions were significantly different between WT and DKO at $72 \mathrm{~h}$ of activation, with substantially more regions gaining $5 \mathrm{hmC}$ in control compared to Tet2/3 DKO B cells at each time point examined (Fig. 2d, 2e). Of 2,139 "TET-regulated" DhmRs with higher 5hmC in WT compared to Tet2/3 DKO B cells ("WT>DKO DhmR”), 2020 (94.4\%) significantly overlapped with DhmR ${ }^{72-u p ~(2020 / 8454, ~}$ 23.9\%). Consistently, "WT>DKO DhmR" were located more than $10 \mathrm{~kb}$ from the TSS (Fig. S2c), showed decreased DNA methylation at their centers (Fig. S2d), and were enriched for NF-KB (Rel homology domain, RHD), bZIP and composite IRF:bZIP motifs (Fig. 2f).

\section{Tet2 and Tet3 regulate immunoglobulin class switch recombination (CSR)}

To assess the effect of Tet2/3 deletion on the antibody response in vivo, we treated Tet2 ${ }^{\text {fl/fl }}$ Tet $3^{\text {fl/fl }}$ Rosa26LSL-YFP Cre ${ }^{E R T 2}$ and control Tet2 ${ }^{f / f l}$ Tet $^{f / f f l}$ Rosa26-LSL-YFP mice for 5 days with tamoxifen, followed by immunization with NP-OVA in the footpads two days later. Germinal center responses in draining popliteal lymph nodes were analyzed by flow cytometry on day 7 post-immunization, gating on $\mathrm{YFP}^{+} \mathrm{B}$ cells in Tet2/3 DKO B cells (Fig. 3a). The overall percentage of germinal center (GC) B cells (CD19 GL7 $^{+}$Fas $^{+}$) was similar between WT and DKO (Fig. 3b, quantified in Fig. 3c), indicating that acute deletion of Tet2/3 had no immediate impact on the formation of GC B cells. The most striking phenotype, however, was the consistent decrease in CSR from $\operatorname{lgM}$ to $\lg \mathrm{G} 1$ in both total and NP-specific germinal center B cells (Fig. 3d-f), demonstrating a role for TET proteins in regulating antibody responses in vivo, particularly the CSR.

To determine if the CSR phenotype was B-cell-intrinsic, B cells from tamoxifen-treated mice were labeled with proliferation dye (Cell-trace Violet) and activated with LPS and IL-4 for 4 days (Fig. 3g). Consistent with the CSR defect in vivo, we noticed a consistent decrease in IgG1 switching in Tet2/3 DKO B cells activated in vitro relative to WT B cells (Fig. 3h, 3i). The impaired CSR in Tet2/3 DKO is not due to Cre activity, as similar result was observed when Cre ${ }^{E R T 2}{\text { Tet } 2^{+/+}}$Tet3 $^{+/+}$Rosa26-LSL-YFP was used as control (Fig. S3a). The defect in CSR was cell-intrinsic, since it was also apparent when congenically-marked WT (CD45.1) and Tet2/3 DKO (CD45.2) B cells were mixed and co-cultured (Fig. S3b). The difference was not due to altered proliferation, which was comparable between WT and Tet2/3 DKO B cells (Fig. 3h, Fig. S3c). Correlating with the decrease in CSR from IgM to IgG1, the expression of circular $\gamma 1$ transcript was decreased in Tet2/3 DKO B cells (Fig. 3j). Further, CSR to IgA was also decreased in Tet2/3 DKO relative to WT B cells activated with anti-CD40, IL4, IL-5, and TGF $\beta$ (Fig. 3k-3n). Notably, reconstitution of Tet2/3 DKO B cells with the enzymatically active catalytic domain of TET2 (Tet2CD) restored CSR from IgM to IgG1 almost to control levels, whereas an 
enzymatically inactive mutant of Tet2CD (Tet2CD ${ }^{\mathrm{H} \times \mathrm{D}}$ ) was ineffective (Fig. S3d-3f). These results indicate that Tet2 and Tet3 are required for optimal CSR both in vitro and in vivo, and that their catalytic activity is required.

Because the CSR defect in Tet2/3 DKO B cells was $~ 50 \%$ of control, we asked whether deletion of all three TET proteins might have a more striking effect. We treated $C r e^{E R T 2}$ Tet $^{f l / f l}$ Tet2 $2^{\text {fl/fl }}$ Tet $3^{\text {fl/fl}}$ Rosa26-LSL-YFP and control Tet $1^{f / f l}$ Tet2 ${ }^{f / f l}$ Tet3 $^{\text {ff/fl }}$ Rosa26-LSL-YFP mice for 5 days with tamoxifen, followed by NP-OVA immunization as in Fig. 3a. Consistent with the very low expression of Tet1 in mature B cells (Fig. 2a), the CSR defect in Tet1/2/3 TKO was comparable to that observed in Tet2/3 DKO mice, with decreased IgG1switched cells (Fig. S3g-3h). These results indicate that Tet2 and Tet3 are the major TET proteins that regulate CSR in $\mathrm{B}$ cells.

\section{Tet2 and Tet3 regulate expression of the cytidine deaminase AID}

CSR is a highly regulated process and involves multiple pathways, including cytokine signaling and DNA repair (35). RNA-seq analysis identified a relatively small number of genes differentially expressed between WT and Tet2/3 DKO B cells under resting conditions and at different time points after activation (Fig. S4a, 4b); among these was Aicda, which encodes AID, the activation-induced cytidine deaminase essential for CSR. qRT-PCR analysis confirmed an $\sim 50 \%$ decrease in Aicda mRNA expression in Tet2/3 DKO relative to WT B cells at each time point from 48 to 96 hours post-activation (Fig. 4a, Fig. S4c), a phenotype reminiscent to the dampened CSR in the case of AID haploinsufficiency $(36,37)$ (Fig. S4d-i). The decrease in protein expression was even more profound, as shown by immunoblotting (Fig. 4b).

To determine if the decrease in AID expression was fully responsible for the CSR defect, we expressed WT and catalytically inactive AID in WT and Tet2/3 DKO B cells via retroviral transduction. Prior to transduction, B cells from WT and DKO mice were stimulated for 24 hours with LPS and $F\left(a b^{\prime}\right)_{2}$ anti-lgM, a stimulation condition in which CSR was inhibited and AID expression was delayed (38). CSR was induced with LPS and IL-4 24h post-transduction. We argued that if Tet2 and Tet3 regulated additional major process(es) downstream of AID, re-expressing AID in Tet2/3 DKO would only have a marginal effect on the observed CSR phenotype. In fact, retroviral expression of catalytically active AID (AID ${ }^{\mathrm{WT}}$ ) largely rescued the CSR defect in Tet2/3 DKO B cells; catalytic activity was required as expression of a catalytically inactive AID mutant (AID ${ }^{\text {MUT }}$ ) had no effect on CSR (bottom panels; Fig. 4c, 4d). Similar to previous observations (12), expression of AID in WT cells also increased the frequency of $\operatorname{lgG} 1^{+}$cells (top left and middle panels; Fig. 4c, 4d). Despite their importance in Aicda expression, Tet2/3 were not required for the expression of $\mu$ and $\gamma 1$ germline transcripts that are essential for CSR (Fig. S4j). These data suggest that the bulk of the CSR defect in Tet2/3 DKO B cells can be attributed to the decrease in expression of Aicda mRNA and AID protein, leading us to test the hypothesis that TET proteins control Aicda expression through distal regulatory element(s) of the Aicda gene.

\section{Identification of TET-responsive regulatory elements in the Aicda locus}

Multiple conserved regulatory elements influence Aicda expression (Fig. S5a), including an intronic region located $26 \mathrm{~kb}$ 5' of the Aicda TSS, within the adjacent Mfap5 gene; two intergenic regions between the Mfap5 and Aicda genes (located at $-21 \mathrm{~kb}$ and $-8 \mathrm{~kb} 5$ ' of the Aicda TSS respectively); a region in the first intron of Aicda; and a region located 3' of the Aicda gene. Deletion of any of the above elements, either in the endogenous locus or in the context of BAC transgenes, dramatically decreased the expression of Aicda in activated $B$ cells (17-20), indicating that they have essential, non-redundant regulatory (enhancer) function and that their concerted action is necessary for Aicda induction. The Aicda 5' enhancer at -26 kb in the Mfap5 gene, the intergenic 5' enhancers, and the intron 1 enhancer noticeably gain H3K27Ac and lose 5mC upon activation, and have been collectively termed the Aicda "superenhancer" (20,33) (Fig. 5a, middle and bottom tracks). 
To determine if these regulatory elements were detectably occupied by Tet2 upon activation, we performed chromatin immunoprecipitation-sequencing (ChIP-seq) for Tet2 in B cells at $72 \mathrm{~h}$ following activation. Indeed, each of these elements was occupied by Tet2 in $72 \mathrm{~h}$-activated B cells (Fig. 5a, top two tracks). Among these, the Mfap5 intronic region and the intergenic region, located at $-26 \mathrm{~kb}$ and $-8 \mathrm{~kb} 5$ ' of the TSS were clearly "TET-regulated": their $5 \mathrm{hmC}$ levels increased after activation of WT B cells, and this increase was significantly diminished in Tet2/3 DKO B cells (Fig. 5b), placing them in the category of WT>DKO DhmRs (Fig. 2d, 2e). We have termed these enhancers TetE1 $(-8 \mathrm{~kb})$ and TetE2 (-26 kb) respectively; TetE1 is potentially the prime target for Tet $2 / 3$ due to its larger gain of $5 \mathrm{hmC}$ after activation (Fig. $\mathbf{5 b}$ ).

To confirm the importance of TetE1 in Aicda regulation, we deleted the enhancer using CRISPR in CH12F3 cells, a B cell line that can class-switch from IgM to IgA upon activation with anti-CD40/IL-4/TGF $\beta$ (Fig. S5b, 5c). We tested four clones with homozygous deletions; all showed decreased expression of Aicda mRNA, and in three of these, there was almost no detectable CSR (Fig. S5d, 5e), confirming a previous report in the context of a BAC transgene that TetE1 was essential for Aicda expression (17).

$B$ cell activation induces strong DNA demethylation (loss of WGBS signal) at TetE1 (Fig. 5a). Since bisulfite sequencing does not distinguish $5 \mathrm{mC}$ and $5 \mathrm{hmC}$, we used oxidative bisulfite sequencing (oxBS-seq) to assess the levels of $5 \mathrm{mC}, 5 \mathrm{hmC}$ and unmodified $\mathrm{C}$ at TetE1 in WT and Tet2/3-DKO cells (neither BS-seq nor oxBSseq distinguish unmodified $\mathrm{C}$ from $5 \mathrm{fC}$ and $5 \mathrm{caC}$, but these modified bases are $\sim 10$-fold and $\sim 100$-fold less abundant than $5 \mathrm{hmC}(2))$. Consistent with the fact that global $5 \mathrm{mC}$ and $5 \mathrm{hmC}$ levels are similar between WT and Tet2/3 DKO B cells prior to activation (Fig. S2b), CpGs in both TetE1 and the Aicda promoter displayed similar levels of $5 \mathrm{mC}$ and $5 \mathrm{hmC}$ prior to activation (Fig. 5c, compare $0 \mathrm{~h}$ panels). At $72 \mathrm{~h}$ following activation, however, there was a substantial increase in both $5 \mathrm{hmC}$ and unmodified $\mathrm{C}$ in WT B cells, whereas Tet2/3 DKO B cells displayed little or no $5 \mathrm{hmC}$ and remained significantly methylated (Fig. 5c, compare $72 \mathrm{~h}$ panels). These results indicate that Tet2 and Tet3 regulate Aicda expression by binding to and depositing $5 \mathrm{hmC}$ at both 5 ' enhancers, TetE1 and TetE2.

\section{Tet2 and Tet3 maintain chromatin accessibility at the Aicda Tet-responsive elements TetE1 and TetE2}

Transcriptional regulatory regions are typically found in accessible regions of chromatin (39), and were shown to be marked by $5 \mathrm{hmC}$ during $\mathrm{T}$ and $\mathrm{B}$ cell development $(4,34)$. To assess the dynamics of changes in chromatin accessibility, we performed ATAC-seq in B cells stimulated with LPS and IL-4. Activated B cells displayed progressive chromatin remodeling, with thousands of regions showing changes in accessibility compared to naïve B cells, ranging from 15,529 differentially accessible regions (DARs) at 24h to 28,106 DARs at $72 \mathrm{~h}$ (Fig. 6a). Regions with increased $5 \mathrm{hmC}$ after activation (DhmR ${ }^{72 \mathrm{~h}-\mathrm{up}}$ ) also showed increased chromatin accessibility following activation, and vice versa (Fig. S6a, blue box-and-whisker plots and not shown).

To understand the relationship between TET function and chromatin accessibility, we performed ATAC-seq on WT and Tet2/3 DKO B cells activated as in Fig. 3g. Of a total of $\sim 28,000$ accessible regions (Fig. 6b, 6c), only a minor fraction ( $1.5 \%$; 421 of 28137$)$ showed significant changes in accessibility between WT and Tet2/3 DKO B cells and the differences were observed late, at 72h following activation (Fig. 6b-d). Of the 292 potentially TET-regulated DARs, defined as showing decreased accessibility in Tet2/3 DKO compared to WT B cells (WT>DKO DARs), the majority were located distal to the TSS (Fig. S6b) and showed a Tet2/3-dependent increase in 5hmC (Fig. 6c, Fig. S6c, upper panels). In contrast, the 129 DKO>WT DARs that were less accessible in WT compared to Tet2/3 DKO B cells, and the 27716 commonly accessible DARs, were present in both TSS-proximal and -distal regions, and did not show significant changes in 5hmC (Fig. 6c, middle and lower panels; Fig. S6b, 6c). Analysis of DNA methylation at 48h post-activation showed that WT>DKO DARs were further demethylated after activation, whereas DKO>WT DARs were already substantially demethylated in naïve $B$ cells and showed no further changes after activation (Fig. S6d). Moreover, WT>DKO DARs resembled DhmR $^{72 h-u p}$ (Fig. 1f) and WT>DKO DhmRs (Fig. 2f) in their enrichment for bZIP and BATF:IRF motifs (Fig. S6e). 
Focusing on the Aicda locus, we found that activation was associated with increased accessibility at the Aicda enhancers TetE1 and TetE2 (Fig. 6d). The $5 \mathrm{hmC}$ modification continuously increased at these two elements until $72 \mathrm{~h}$, with a higher level of deposition at TetE1 (see Fig. 5b). In contrast, the time course of increase in chromatin accessibility was quite different at the two enhancers (Fig. 6d): TetE2 showed a rapid increase in accessibility apparent in both WT and Tet2/3 DKO B cells at $24 \mathrm{~h}$ following activation, whereas the time course of increase in TetE1 accessibility was slower, matching that of $5 \mathrm{hmC}$ deposition (compare Fig.s $\mathbf{5 b}$ and $\mathbf{6 d}$ ). Consistent with the increased accessibility, several chromatin remodelers and histone acetyltransferases including Brg1, Chd4, p300, and to a lesser extent, Gcn5, were recruited to TetE1 and TetE2 in $24 \mathrm{~h}$ activated $\mathrm{B}$ cells (Fig. S6f). Interestingly, we noticed a slight decrease in chromatin accessibility at TetE1 and TetE2 in Tet2/3 DKO B cells compared with WT cells at 72 hours, suggesting that TET proteins are important for maintaining the accessibility at these enhancers (Fig. 6e). Together these data point to a consistent link between bZIP-family transcription factors, TET catalytic activity and chromatin accessibility that is explored in greater detail below

\section{Batf acts upstream of TET at Aicda enhancers}

Before enhancers are established during development, cell lineage specification or activation, certain key transcription factors bind to nucleosome-associated regions and recruit chromatin remodelling complexes and histone modifying enzymes to create active enhancers (32). Chromatin remodelling complexes establish accessible (nucleosome-depleted) regions, while the histone acetyltransferase p300/CBP deposits the "active" histone modification H3K27Ac (32). To identify potential pioneer transcription factors for the Aicda locus, we took advantage of our previous motif enrichment analyses (Fig.s 1f, 2f; Fig. S1g). We had observed strong enrichment for consensus binding motifs for bZIP transcription factors, at regions that progressively gained $5 \mathrm{hmC}$ as a function of activation (DhmR $\left.{ }^{\text {up }}, \mathbf{F i g} . \mathbf{1 f}\right)$, regions that lost DNA methylation upon activation (DMR ${ }^{48 \mathrm{~h}-}$ down; Fig. S1g), regions with higher $5 \mathrm{hmC}$ in WT compared to Tet2/3 DKO B cells (WT>DKO DhmRs, Fig. 2f), and regions with higher accessibility in WT versus Tet2/3 DKO B cells (DAR ${ }^{72 h}$ WT>DKO; Fig. S6e).

Based on these data, we focused on bZIP transcription factors expressed in activated B cells. Consistent with previous observations $(40,41)$, Batf was expressed at low levels prior to activation but was highly expressed from $24 \mathrm{~h}$ to $96 \mathrm{~h}$ following activation (Fig. 7a). This increase in Batf expression preceded that of Aidca, as expected if Batf regulated Aidca mRNA induction (Fig. S7a). In contrast, expression of Bach1 and AP-1 (Fos and Jun) family members was either low throughout (Fos/1, Fos/2, JunD, Bach1), or moderate to high in unstimulated B cells and strongly reduced by $24 \mathrm{~h}$ (Fos, FosB, Jun, JunB) or $72 \mathrm{~h}$ (Bach2) of activation (Fig. S7b-d). Given the kinetics, we examined the importance of Batf in subsequent experiments.

Batf is essential for controlling both $\mathrm{T}$ and $\mathrm{B}$ cell function during humoral responses $(40,41)$. Importantly, Batf-KO B cells are defective in CSR (Fig. S7e) (40,41), thus resembling Tet2/3 DKO B cells (Fig. 3). Genome-wide analysis of Batf binding by ChIP-seq in 72h-activated WT and Tet2/3-DKO B cells showed very few overall differences (Fig. S7f), indicating that Batf functioned upstream or independently of TET enzymes. Nevertheless, one of two distinguishable sets of Batf ChIP-seq peaks (Cluster 2 in Fig. 7b; n=9,442) was clearly TET-regulated, since the peaks in this cluster showed a progressive Tet2/3-dependent increase in $5 \mathrm{hmC}$ with time after activation (Fig. $\mathbf{7 b}, \mathbf{c})$. In contrast, the majority of Batf peaks in Cluster $1(\mathrm{n}=2,198)$ showed no significant activation-dependent increase in $5 \mathrm{hmC}$ (Fig. 7b; upper panel).

Batf bound strongly at the TetE1 and TetE2 enhancers in the Aicda locus, and to a lesser extent to the $-21 \mathrm{~kb}$ intergenic enhancer located between TetE1 and TetE2 (Fig. 7d, top tracks). This binding pattern resembles that of Tet2 (Fig. 5a), as well as that of E2A and PU.1 (Fig. 7d), three transcriptional regulators already expressed in unstimulated B cells (Fig. S7g, 7h; Fig. 2a)(21, 42, 43). Moreover, BATF as well as JUNB associated with TetE1 and TetE2 in a human B cell lymphoblast (Fig. S7i), suggesting the binding of BATF is evolutionarily conserved. To determine whether Batf acted upstream of TET proteins (for instance by recruiting Tet2 and Tet3 to Batf-bound regulatory regions), we analysed $5 \mathrm{hmC}$ deposition at the Batf- and TET- 
responsive element TetE1 in WT and Batf-deficient B cells. We found unambiguously that the absence of Batf abolished activation-induced hydroxymethylation at TetE1 (Fig. 7e), indicating that Batf is required for TETmediated hydroxymethylation at this Aicda enhancer. Our results are consistent with the hypothesis that Batf recruits Tet2 and/or Tet3 to TetE1 and TetE2 and facilitates Aicda expression by promoting $5 \mathrm{hmC}$ modification and DNA demethylation at these upstream Aicda enhancers. 


\section{Discussion}

TET proteins (TET1, TET2 and TET3) oxidize $5 \mathrm{mC}$ to $5 \mathrm{hmC}$, a stable epigenetic mark that is the most abundant of the three oxi-mC intermediates for DNA demethylation. Due to the pleiotropic effects of TET proteins in cells, it has been challenging to address the specific roles of TET proteins in mice with prolonged TET deficiency. Here, to circumvent this issue, we used the inducible tamoxifen-Cre ${ }^{E R T 2}$ system to delete Tet2 and Tet3 in mature B cells, a well-established system for the molecular analysis of gene regulation during cell activation. Our data show clearly that Tet2 and Tet3 - the major TET proteins expressed in B cells - are required for efficient class switch recombination (CSR) both in vivo and in cultured cells. A primary mechanism involves TET-mediated regulation of the expression of Aicda, the essential DNA cytosine deaminase for CSR. The activation-induced transcription factor BATF, potentially with other transcription factors, recruits TET proteins to two major TET-responsive regulatory elements that we have newly defined in the Aicda locus, TetE1 and TetE2. Tet2 and Tet3 convert $5 \mathrm{mC}$ to $5 \mathrm{hmC}$ at these regulatory elements, leading to DNA demethylation, sustaining enhancer accessibility and augmenting Aicda expression.

The biological consequences of TET loss-of-function are determined by several factors: the time course of Tet2 and Tet3 gene deletion, the stability of Tet2 and Tet3 mRNA and protein, and the rate of cell division which determines the rate of passive (i.e. replication-dependent) dilution of $5 \mathrm{hmC}$. At each cell division, hemimethylated $\mathrm{CpGs}$ are recognised by the maintenance UHRF1/DNMT1 DNA methyltransferase complex and converted back to symmetrically methylated $\mathrm{CpGs}$, whereas hemi-hydroxymethylated $\mathrm{CpGs}$ are ignored and so are diluted by half $(1,2)$. Consequently, $5 \mathrm{hmC}$ is present at comparable levels in quiescent (non-dividing) WT and Tet2/3 DKO B cells, thus enabling us to study the effects of acute TET deletion in activated, proliferating B cells. The progressive replication-dependent loss of $5 \mathrm{hmC}$ and consequent dilution of both $5 \mathrm{mC}$ and $5 \mathrm{hmC}$ is likely to be required for optimal gene expression, explaining the long-standing observation that the induction of Aicda expression during B cell activation, and the induction of cytokine genes during Th2 differentiation, are both tightly coupled to cell division $(22,44)$.

An optimal level of AID is crucial to maintain the necessary balance between effective antibody immune responses and unintentional $\mathrm{C}>\mathrm{T}$ mutations caused by AID-mediated DNA cytidine deamination. Indeed, while Aicda haploinsufficiency results in dampened antibody responses $(36,37$ ) (Fig. S4e-j), uncontrolled AICDA expression is associated with B cell malignancies (45). Thus, the level and activity of AID are meticulously controlled by diverse mechanisms including a tight transcriptional regulatory program (16). At the Aicda locus, at least six regulatory elements have been identified (Fig. S5a); five of them, located at distances ranging from -29 to $+5 \mathrm{~kb}$ relative to the TSS, are collectively termed the Aicda superenhancer $(20,33)$. The enhancers at $26,-21,-8,+13 \mathrm{~kb}$ are all necessary for inducing Aicda expression in activated $\mathrm{B}$ cells, based on deletion of individual enhancers in mice and the $\mathrm{CH} 12 \mathrm{~B}$ cell line $(17,18)$. Notably, even in naïve B cells where Aicda is not expressed, the Aicda promoter is already highly enriched in $5 \mathrm{hmC}$ and the -21 , intronic, $+13 \mathrm{~kb}$ Aicda enhancers display 5hmC and H3K27Ac (Fig. S5a). In fact, the 5hmC modification at the $-26,-21,+13 \mathrm{~kb}$ Aicda enhancers is apparent as early as the pro-B cell stage of B cell development (4), suggesting that TETmediated $5 \mathrm{hmC}$ modification acts to "bookmark" regulatory elements necessary for proper gene expression in progeny cells after activation.

The vast majority of $5 \mathrm{hmC}$-marked regions are present in common between naïve and activated mature $B$ cells (this study), and between WT and TET-deficient iNKT cells (34), supporting the hypothesis that most $5 \mathrm{hmC}$-marked regions in any given cell type were laid down during prior developmental stages and thus are constitutively modified. In contrast, activation-induced $5 \mathrm{hmC}$ modification occurs at only a few distal elements in B cells (Fig. 1c), and $5 \mathrm{hmC}$ levels at these elements correlate strongly with activation-induced increases in enhancer activity defined by H3K27Ac (Fig. 1g) (46). Moreover, the majority of previously described B cell superenhancers $(20,33)$ harbor at least one activation-induced, $5 \mathrm{hmC}$-modified regulatory element (Fig. 1h). In the particular case of Aicda, we identified activation-induced $5 \mathrm{hmC}$ modification at two major TETresponsive elements, TetE1 and TetE2, both part of a superenhancer cluster located 5'of the Aicda gene (Fig. 
5a) $(20,33)$. $5 \mathrm{hmC}$ modification at these elements was apparent by $48 \mathrm{~h}$ (Fig. 5b), preceding the dramatic upregulation of Aicda mRNA at $72 \mathrm{~h}$ (Fig. S4c). Loss of Tet2 and Tet3 almost eliminated the induced 5hmC modification at both enhancers and resulted in diminished expression of both Aicda mRNA and AID protein (Fig. 4a, 4b, 5b; Fig. S4c), suggesting that TET proteins and $5 \mathrm{hmC}$ are required for the TetE1 and TetE2 enhancers to function at full capacity.

Studies from our lab and others have implicated TET proteins and $5 \mathrm{hmC}$ in regulating chromatin accessibility. For instance, TET proteins were shown to be required for demethylation of evolutionarily conserved enhancers during zebrafish development, and morpholino-mediated knockdown of Tet1/2/3 resulted in decreased enhancer accessibility (47). In mammals, we have shown that Tet2/3-deficiency results in lower accessibility of enhancers during $T$ and $B$ cell development $(4,34)$. However, these steady state studies provide limited mechanistic insights. Here, through systematic analyses of $5 \mathrm{hmC}$ modification and chromatin accessibility kinetics during $B$ cell activation, we show that $5 \mathrm{hmC}$ displays a time-dependent increase at regions that are differentially accessible between WT and Tet2/3 DKO B cells during; moreover TET proteins are important for sustaining enhancer accessibility (Fig. 6e; Fig. S6e). We speculate that enhancer methylation limits enhancer output through recruitment of repressive complexes associated with a variety of proteins that bind methylcytosine or methylated CpGs (48), and that TET-mediated CpG hydroxymethylation and subsequent DNA demethylation are required to maintain enhancer accessibility, perhaps through recruitment of CXXC domain proteins such as Cpf1, a component of the SETD1 H3K4 methyltransferase complex (49).

How the activity of TET proteins is directed to specific sites in the genome remains an open question. In a previous study, we showed that TET proteins are recruited to lg light chain enhancers during $B$ cell development, potentially via the transcription factors PU.1 and E2A, and documented direct protein-protein interactions of Tet2 with both E2A and PU.1 (4). Here we propose that promiscuous interaction with transcription factors is a general mechanism for recruitment of TET proteins to specific loci, similar to the known interactions of p300 with hundreds of transcription factors (50). To identify the transcription factors mediating TET recruitment in activated B cells, we took advantage of our genome-wide analyses to assess enrichment for consensus transcription factor binding motifs in multiple datasets (5hmC, ATAC and DNA methylation). We found a consistent enrichment for NFKB, bZIP and bZIP:IRF composite binding motifs (Fig.s 1f, 2f; Fig. S1g, 6e). Based on a recent study showing that AP-1 (bZIP) family proteins, together with lineagedetermining transcription factors, are "pioneer" factors capable of binding nucleosomal DNA and recruiting the BAF (SWI/SNF) complex for nucleosome remodeling (51), we focused on transcription factors of the bZIP family.

Our data suggest strongly that the bZIP transcription factor Batf is one of the major bZIP transcription factors responsible for TET recruitment to the Aicda locus. Batf is strongly induced at the mRNA level prior to Aicda induction in activated B cells (Fig. 7a), and Batf deficiency in B cells is associated with a dramatic impairment of AID expression and CSR $(40,41)$. Although loss of Tet2 and Tet3 had no significant effect on global Batf binding (Fig. 7b; Fig. S7d), Batf was required for 5hmC modification at TetE1 (Fig. 7e). Composite bZIP:IRF motifs and AP-1 motifs that support BATF:JUN:IRF and BATF:JUN cooperation respectively were enriched in our genome-wide 5hmC, ATAC and DNA methylation datasets (Fig. 1f, 2f; Fig. S1g, 6e), consistent with previous findings that B cells lacking BATF, and IRF4/IRF8 show impaired Aicda induction and CSR (40, 41, 52-54). We propose that together, BATF:JunB and BATF:IRF assemblies recruit TET proteins as well as chromatin remodelling complexes to diverse enhancers including the Aicda enhancers TetE1 and TetE2 in activated B cells, thereby promoting enhancer accessibility, $\mathrm{hmC}$ deposition and DNA demethylation.

Our data emphasize the utility of $5 \mathrm{hmC}$ mapping for easy, one-step analysis of transcriptional and epigenetic landscapes in any cell type of interest. $5 \mathrm{hmC}$ is a quintessential epigenetic modification that marks the most highly enriched at active enhancers and the gene bodies of highly transcribed genes, and the relative levels of $5 \mathrm{hmC}$ at enhancers and gene bodies provide good estimates of enhancer function and the magnitude of 
transcription respectively (46). 5hmC mapping by CMS-IP sufficed to identify all known enhancers in the Aicda locus, in a manner that was superior to both H3K27Ac and Tet2 ChIP-seq, and changes in 5hmC identified enhancers relevant to any particular process of cell activation or differentiation separately from all enhancers in the genome. Given its high chemical stability, the fact that its measurement requires only purified DNA, and the availability of methods for its sensitive and specific detection, $5 \mathrm{hmC}$ is an appealing epigenetic mark for studying gene regulation. Overall, $5 \mathrm{hmC}$ distribution contains information analogous to those from ATAC-seq and ChIP-seq for enhancer histone modifications, effectively providing a transcriptional history of any given cell type written in DNA. If the genome is akin to an encyclopedia, $5 \mathrm{hmC}$ highlights those entries most relevant to a particular biological process.

\section{Acknowledgements}

We would like to thank you Dr. Uttiya Basu for providing the AID antibody; Dr. Kefei Yu providing the AicdaKO CH12F3 cells; Dr. Paolo Casali and Dr. Hong Zan for discussion; Laura Hempleman for assisting animal experiments; Cheryl Kim, Lara Nosworthy, Denise Hinz, and Robin Simmons (LJI Flow Cytometry Core) for help with cell sorting; Jeremy Day and Nick Wlodychak (LJI Functional Genomics Center) for assistance with next generation sequencing. C.-W.J.L. was supported by a Cancer Research Institute Irvington Postdoctoral Fellowship. V.S. is supported by Leukemia and Lymphoma Society Postdoctoral Fellowship. A.R. is supported by the National Institutes of Health (NIH) grants R35 CA210043 and R01 Al109842. D.G.S. supported by NIH Grant R01 Al127642. F.A. and A.C. have been partially supported by Institute Leadership Funds from La Jolla Institute for Allergy and Immunology and by the NIH Grant R01 MH111267. Funding for Illumina HiSeq 2500 and BD FACSAria II is supported by NIH (NIH S10OD016262, NIH S10RR027366).

\section{Author Contributions}

C.-W.J.L. and V.S. conceptualized the experiments, acquired, analyzed the data, and wrote the manuscript. D.S.C. and E.G.A. performed majority of bioinformatics analysis (ATAC-seq, CMS-IP, enhancers analysis, WGBS, oxBS-seq, ChIP-seq), and proofread the manuscript. A.C. performed and advised on bioinformatics analysis (RNA-seq, TC-seq). C.-W.J.L. performed bioinformatics analysis (motifs, region characterization, data visualization) and the initial exploratory analysis. X.Y. provided key reagents and assistance for experiment (oxBS-seq). F.A. supervised the bioinformatics analysis and reviewed the manuscript. D.G.S. provided initial advised, key reagents, data interpretation, and reviewed the manuscript. A.R. supervised, conceptualized the experiments, data interpretation, and wrote the manuscript.

\section{Declaration of Interests}

The Authors declare no competing interests. 
bioRxiv preprint doi: https://doi.org/10.1101/438531; this version posted October 8,2018 . The copyright holder for this preprint (which was not certified by peer review) is the author/funder. All rights reserved. No reuse allowed without permission.

\section{Supplementary Tables}

Supplementary Table S1.

Differentially expressed genes between WT and Tet2/3-DKO B cells, related to Fig. S4.

Supplementary Table S2.

Time-series analysis of RNA expression in WT B cells, related to Fig. S7.

Supplementary Table S3.

Primers sequences.

Supplementary Table S4.

Reagents and resource. 


\section{References}

1. X. Wu, Y. Zhang, TET-mediated active DNA demethylation: mechanism, function and beyond. Nature reviews. Genetics 18, 517 (Sep, 2017).

2. A. Tsagaratou, C. J. Lio, X. Yue, A. Rao, TET Methylcytosine Oxidases in T Cell and B Cell Development and Function. Frontiers in immunology 8, 220 (2017).

3. J. P. Scott-Browne, C. W. Lio, A. Rao, TET proteins in natural and induced differentiation. Current opinion in genetics \& development 46, 202 (Oct, 2017).

4. C. J. Lio et al., Tet2 and Tet3 cooperate with B-lineage transcription factors to regulate DNA modification and chromatin accessibility. Elife 5, (Nov 21, 2016).

5. S. Orlanski et al., Tissue-specific DNA demethylation is required for proper B-cell differentiation and function. Proceedings of the National Academy of Sciences of the United States of America 113, 5018 (May 3, 2016).

6. C. Quivoron et al., TET2 inactivation results in pleiotropic hematopoietic abnormalities in mouse and is a recurrent event during human lymphomagenesis. Cancer cell 20, 25 (Jul 12, 2011).

7. R. Schmitz et al., Genetics and Pathogenesis of Diffuse Large B-Cell Lymphoma. The New England journal of medicine 378, 1396 (Apr 12, 2018).

8. A. Reddy et al., Genetic and Functional Drivers of Diffuse Large B Cell Lymphoma. Cell 171, 481 (Oct 5, 2017).

9. N. S. De Silva, U. Klein, Dynamics of B cells in germinal centres. Nature reviews. Immunology 15, 137 (Mar, 2015).

10. V. Chandra, A. Bortnick, C. Murre, AID targeting: old mysteries and new challenges. Trends in immunology 36, 527 (Sep, 2015).

11. B. Vaidyanathan, J. Chaudhuri, Epigenetic Codes Programing Class Switch Recombination. Frontiers in immunology 6, 405 (2015).

12. M. Muramatsu et al., Class switch recombination and hypermutation require activation-induced cytidine deaminase (AID), a potential RNA editing enzyme. Cell 102, 553 (Sep 1, 2000).

13. F. W. Alt, Y. Zhang, F. L. Meng, C. Guo, B. Schwer, Mechanisms of programmed DNA lesions and genomic instability in the immune system. Cell 152, 417 (Jan 31, 2013).

14. R. Casellas et al., Mutations, kataegis and translocations in B cells: understanding AID promiscuous activity. Nature reviews. Immunology 16, 164 (Mar, 2016).

15. D. F. Robbiani, M. C. Nussenzweig, Chromosome translocation, B cell lymphoma, and activation-induced cytidine deaminase. Annual review of pathology 8, 79 (Jan 24, 2013).

16. H. Zan, P. Casali, Epigenetics of Peripheral B-Cell Differentiation and the Antibody Response. Frontiers in immunology 6, 631 (2015).

17. T. Huong le et al., In vivo analysis of Aicda gene regulation: a critical balance between upstream enhancers and intronic silencers governs appropriate expression. PloS one 8, e61433 (2013).

18. T. H. Tran et al., B cell-specific and stimulation-responsive enhancers derepress Aicda by overcoming the effects of silencers. Nature immunology 11, 148 (Feb, 2010).

19. E. E. Crouch et al., Regulation of AID expression in the immune response. The Journal of experimental medicine 204, 1145 (May 14, 2007).

20. K. R. Kieffer-Kwon et al., Interactome maps of mouse gene regulatory domains reveal basic principles of transcriptional regulation. Cell 155, 1507 (Dec 19, 2013).

21. M. Wohner et al., Molecular functions of the transcription factors E2A and E2-2 in controlling germinal center $B$ cell and plasma cell development. The Journal of experimental medicine 213, 1201 (Jun 27, 2016).

22. J. S. Rush, M. Liu, V. H. Odegard, S. Unniraman, D. G. Schatz, Expression of activation-induced cytidine deaminase is regulated by cell division, providing a mechanistic basis for division-linked class switch recombination. Proceedings of the National Academy of Sciences of the United States of America 102, 13242 (Sep 13, 2005).

23. K. R. Kieffer-Kwon et al., Myc Regulates Chromatin Decompaction and Nuclear Architecture during B Cell Activation. Molecular cell 67, 566 (Aug 17, 2017).

24. Y. Huang, W. A. Pastor, J. A. Zepeda-Martinez, A. Rao, The anti-CMS technique for genome-wide mapping of 5hydroxymethylcytosine. Nature protocols 7, 1897 (Oct, 2012).

25. W. A. Pastor et al., Genome-wide mapping of 5-hydroxymethylcytosine in embryonic stem cells. Nature 473, 394 (May 19, 2011).

26. W. A. Pastor, L. Aravind, A. Rao, TETonic shift: biological roles of TET proteins in DNA demethylation and transcription. Nature reviews. Molecular cell biology 14, 341 (Jun, 2013).

27. Y. Huang et al., The behaviour of 5-hydroxymethylcytosine in bisulfite sequencing. PloS one 5, e8888 (2010).

28. X. Yue et al., Control of Foxp3 stability through modulation of TET activity. The Journal of experimental medicine 213, 377 (Mar 7, 2016).

29. P. Li et al., BATF-JUN is critical for IRF4-mediated transcription in T cells. Nature 490, 543 (Oct 25, 2012).

30. E. Glasmacher et al., A genomic regulatory element that directs assembly and function of immune-specific AP-1IRF complexes. Science (New York, N.Y.) 338, 975 (Nov 16, 2012). 
31. T. L. Murphy, R. Tussiwand, K. M. Murphy, Specificity through cooperation: BATF-IRF interactions control immune-regulatory networks. Nature reviews. Immunology 13, 499 (Jul, 2013).

32. E. Calo, J. Wysocka, Modification of enhancer chromatin: what, how, and why? Molecular cell 49, 825 (Mar 7, 2013).

33. F. L. Meng et al., Convergent transcription at intragenic super-enhancers targets AID-initiated genomic instability. Cell 159, 1538 (Dec 18, 2014).

34. A. Tsagaratou et al., TET proteins regulate the lineage specification and TCR-mediated expansion of iNKT cells. Nature immunology, (Nov 21, 2016).

35. S. P. Methot, J. M. Di Noia, Molecular Mechanisms of Somatic Hypermutation and Class Switch Recombination. Advances in immunology 133, 37 (2017).

36. I. V. Sernandez, V. G. de Yebenes, Y. Dorsett, A. R. Ramiro, Haploinsufficiency of activation-induced deaminase for antibody diversification and chromosome translocations both in vitro and in vivo. PloS one 3, e3927 (2008).

37. M. Takizawa et al., AID expression levels determine the extent of cMyc oncogenic translocations and the incidence of B cell tumor development. The Journal of experimental medicine 205, 1949 (Sep 1, 2008).

38. H. H. Jabara et al., B-cell receptor cross-linking delays activation-induced cytidine deaminase induction and inhibits class-switch recombination to IgE. The Journal of allergy and clinical immunology 121, 191 (Jan, 2008).

39. C. Roadmap Epigenomics et al., Integrative analysis of 111 reference human epigenomes. Nature 518, 317 (02/18/online, 2015).

40. W. Ise et al., The transcription factor BATF controls the global regulators of class-switch recombination in both B cells and T cells. Nature immunology 12, 536 (Jun, 2011).

41. B. C. Betz et al., Batf coordinates multiple aspects of $B$ and $T$ cell function required for normal antibody responses. The Journal of experimental medicine 207, 933 (May 10, 2010).

42. S. N. Willis et al., Environmental sensing by mature B cells is controlled by the transcription factors PU.1 and SpiB. Nature communications 8, 1426 (Nov 10, 2017).

43. R. Gloury et al., Dynamic changes in Id3 and E-protein activity orchestrate germinal center and plasma cell development. The Journal of experimental medicine 213, 1095 (May 30, 2016).

44. J. J. Bird et al., Helper T cell differentiation is controlled by the cell cycle. Immunity 9, 229 (Aug, 1998).

45. M. Compagno et al., Phosphatidylinositol 3-kinase delta blockade increases genomic instability in B cells. Nature 542, 489 (Feb 23, 2017).

46. A. Tsagaratou et al., Dissecting the dynamic changes of 5-hydroxymethylcytosine in T-cell development and differentiation. Proceedings of the National Academy of Sciences of the United States of America 111, E3306 (Aug 12, 2014).

47. O. Bogdanovic et al., Active DNA demethylation at enhancers during the vertebrate phylotypic period. Nature genetics 48, 417 (Apr, 2016).

48. A. K. Ludwig, P. Zhang, M. C. Cardoso, Modifiers and Readers of DNA Modifications and Their Impact on Genome Structure, Expression, and Stability in Disease. Frontiers in genetics 7, 115 (2016).

49. H. K. Long, N. P. Blackledge, R. J. Klose, ZF-CxxC domain-containing proteins, $\mathrm{CpG}$ islands and the chromatin connection. Biochemical Society transactions 41, 727 (Jun, 2013).

50. H. J. Dyson, P. E. Wright, Role of Intrinsic Protein Disorder in the Function and Interactions of the Transcriptional Coactivators CREB-binding Protein (CBP) and p300. The Journal of biological chemistry 291, 6714 (Mar 25, 2016).

51. T. Vierbuchen et al., AP-1 Transcription Factors and the BAF Complex Mediate Signal-Dependent Enhancer Selection. Molecular cell 68, 1067 (Dec 21, 2017).

52. R. Sciammas et al., Graded expression of interferon regulatory factor-4 coordinates isotype switching with plasma cell differentiation. Immunity 25, 225 (Aug, 2006).

53. U. Klein et al., Transcription factor IRF4 controls plasma cell differentiation and class-switch recombination. Nature immunology 7, 773 (Jul, 2006).

54. C. H. Lee et al., Regulation of the germinal center gene program by interferon (IFN) regulatory factor 8/IFN consensus sequence-binding protein. The Journal of experimental medicine 203, 63 (Jan 23, 2006).

55. M. Ko et al., TET proteins and 5-methylcytosine oxidation in hematological cancers. Immunological reviews 263, 6 (Jan, 2015).

56. M. Ko et al., Ten-Eleven-Translocation 2 (TET2) negatively regulates homeostasis and differentiation of hematopoietic stem cells in mice. Proceedings of the National Academy of Sciences of the United States of America 108, 14566 (Aug 30, 2011).

57. S. Picelli et al., Full-length RNA-seq from single cells using Smart-seq2. Nature protocols 9, 171 (Jan, 2014).

58. F. Ramirez et al., deepTools2: a next generation web server for deep-sequencing data analysis. Nucleic acids research 44, W160 (Jul 8, 2016).

59. Y. Xi, W. Li, BSMAP: whole genome bisulfite sequence MAPping program. BMC bioinformatics 10, 232 (Jul 27, 2009).

60. S. Heinz et al., Simple combinations of lineage-determining transcription factors prime cis-regulatory elements required for macrophage and B cell identities. Molecular cell 38, 576 (May 28, 2010). 
61. M. D. Robinson, D. J. McCarthy, G. K. Smyth, edgeR: a Bioconductor package for differential expression analysis of digital gene expression data. Bioinformatics 26, 139 (Jan 1, 2010).

62. E. P. Consortium, An integrated encyclopedia of DNA elements in the human genome. Nature 489, 57 (Sep 6, 2012).

63. J. D. Buenrostro, P. G. Giresi, L. C. Zaba, H. Y. Chang, W. J. Greenleaf, Transposition of native chromatin for fast and sensitive epigenomic profiling of open chromatin, DNA-binding proteins and nucleosome position. Nature methods 10, 1213 (Dec, 2013).

64. L. Chavez et al., Computational analysis of genome-wide DNA methylation during the differentiation of human embryonic stem cells along the endodermal lineage. Genome research 20, 1441 (Oct, 2010).

65. T. Aijo, X. Yue, A. Rao, H. Lahdesmaki, LuxGLM: a probabilistic covariate model for quantification of DNA methylation modifications with complex experimental designs. Bioinformatics 32, i511 (Sep 1, 2016).

66. X. Lin et al., BSeQC: quality control of bisulfite sequencing experiments. Bioinformatics 29, 3227 (Dec 15, 2013).

67. Q. Song et al., A reference methylome database and analysis pipeline to facilitate integrative and comparative epigenomics. PloS one 8, e81148 (2013).

68. D. Kim, B. Langmead, S. L. Salzberg, HISAT: a fast spliced aligner with low memory requirements. Nature methods 12, 357 (Apr, 2015).

69. D. Kim et al., TopHat2: accurate alignment of transcriptomes in the presence of insertions, deletions and gene fusions. Genome biology 14, R36 (Apr 25, 2013).

70. S. Anders, P. T. Pyl, W. Huber, HTSeq--a Python framework to work with high-throughput sequencing data. Bioinformatics 31, 166 (Jan 15, 2015).

71. J. T. Leek, svaseq: removing batch effects and other unwanted noise from sequencing data. Nucleic acids research 42, (Dec 1, 2014).

72. M. Wu, L. Gu, TCseq: Time course sequencing data analysis. $R$ package version 1.4.0., (2018). 


\section{Figure Legends}

Figure 1. Dynamic changes in $5 \mathrm{hmC}$ during $B$ cell activation.

(a) Flow-chart of experiments. B cells were activated with LPS+IL-4 for the indicated times prior to genome-wide analyses.

(b) Of a total of $159,3055 \mathrm{hmC}$-enriched regions in B cells activated for $72 \mathrm{~h}$, while most regions (grey, $94.1 \%$ ) display similar $5 \mathrm{hmC}$ levels, 8,454 (blue, 5.3\%) show increased $5 \mathrm{hmC}$ and 928 (red, 0.6\%) show decreased $5 \mathrm{hmC}$ relative to naïve $B$ cells. Note that 193 regions represented only in naïve $B$ cells are not shown.

(c) Number of differential hydroxymethylated region (DhmR) showing increased and decreased $5 \mathrm{hmC}$ at respective time points after activation, of a total number of $\sim 160,0005 \mathrm{hmC}$-marked regions present in naïve and activated $B$ cells (Fig. S1a).

(d) Heatmaps showing the kinetics of $5 \mathrm{hmC}$ in the 8,454 regions with increased $5 \mathrm{hmC}$ at $72 \mathrm{~h}$ compared to naïve $\mathrm{B}$ cells (left panels), but no increase in the same number of $5 \mathrm{hmC}$-marked regions common to naïve and $72 \mathrm{~h}$ activated B cells (middle panels). Right panels, only a small number of random genomic regions are marked with $5 \mathrm{hmC}$. For a similar analysis of the 1,121 regions that lose $5 \mathrm{hmC}$ after $72 \mathrm{~h}$ of B cell activation, see Fig. S1c. $5 \mathrm{hmC}$ enrichment is shown as normalized reads per $100 \mathrm{bp}$ bin.

(e) The 85 and 1,953 regions with increased $5 \mathrm{hmC}$ in $24 \mathrm{~h}$ - and $48 \mathrm{~h}$ - activated B cells relative to naïve $B$ cells show decreased "methylation" (bisulfite-resistant cytosine, $5 \mathrm{mC}+5 \mathrm{hmC}$ ) at their centers $48 \mathrm{~h}$ after activation. Average methylation was calculated for each $200 \mathrm{bp}$ bin across $6 \mathrm{~kb}$.

(f) Significant enrichment for consensus RHD (NFKB), IRF:bZIP, and bZIP transcription factor binding motifs in 8,454 regions $\mathrm{DhmR}^{72 \mathrm{~h} \text {-up }}$ showing increased $5 \mathrm{hmC}$ in $72 \mathrm{~h}$-activated relative to naïve $\mathrm{B}$ cells. Common $5 \mathrm{hmC}$ enriched regions were used as background for analysis. Y-axis indicates the fold enrichment versus background, circle size indicates the percentage of regions containing the respective motif, and the color indicates the significance $\left(\log _{10} p\right.$ vaule).

(g) $5 \mathrm{hmC}$ is enriched at active $\left(\mathrm{H} 3 \mathrm{~K} 4 \mathrm{me} 1^{+} \mathrm{H} 3 \mathrm{~K} 27 \mathrm{Ac} \mathrm{c}^{\mathrm{hi}}\right)$ relative to poised $\left(\mathrm{H} 3 \mathrm{~K} 4 \mathrm{me} 1^{+} \mathrm{H} 3 \mathrm{~K} 27 \mathrm{Ac} \mathrm{c}^{\mathrm{lo}}\right)$ enhancers in both activated and naïve $B$ cells. $Y$ and $X$ axes indicate the levels $\left(\log _{2}\right)$ of $H 3 K 4 m e 1$ and $H 3 K 27 A c$ relative to input, respectively.

(h) A substantial fraction of super-enhancers $(76.7 \%, 352$ of 459$)$ identified by high H3K27Ac enrichment overlap with $\mathrm{DhmR}^{72 \mathrm{~h} \text {-up }}$ at which $5 \mathrm{hmC}$ is increased in activated $(72 \mathrm{~h})$ relative to naïve B cells. Fisher exact test was used to analyze the significance. ${ }^{* *}, p<0.01\left(p=8.9203 \times 10^{-266}\right)$. n.s., not significant.

(i) Genome browser view of the Ccr4 locus (mm10; chr9:114,484,000-114,501,000) as an example of a genomic region marked by increased $5 \mathrm{hmC}$, increased $\mathrm{H} 3 \mathrm{~K} 27 \mathrm{Ac}$ and decreased $\mathrm{CpG}$ methylation in activated compared to naïve $B$ cells. Red track indicates $C p G s$ that were included for analysis based on coverage.

(j) Kinetics of increase in Ccr4 mRNA expression (by RNA-seq) in activated B cells. See also Supplementary Fig. 1.

\section{Figure S1, related to Fig. 1. Tet-mediated DNA hydroxymethylation correlates with demethylation and} enhancer activity.

(a) Similar total numbers of $5 \mathrm{hmC}$-enriched regions between naïve and activated $\mathrm{B}$ cells.

(b) Box-and-whisker plot showing the differential hydroxymethylated regions (DhmRs) in activated vs naïve B cells

(see Fig. 1c) are located on average more than $10 \mathrm{~kb}$ from the closest transcription start site (TSS).

(c) Heatmaps showing the kinetics of $5 \mathrm{hmC}$ modification at the 1,121 regions with decreased $5 \mathrm{hmC}$ in $72 \mathrm{~h}$-activated

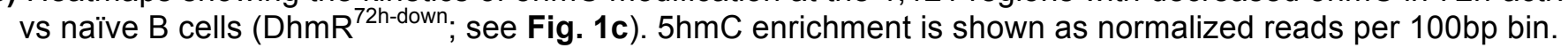

(d) Left, the vast majority of differentially methylated regions (DMRs) with altered WGBS signal $(5 \mathrm{mC}+5 \mathrm{hmC})$ in naïve vs $48 \mathrm{~h}$-activated B cells show decreased DNA methylation. Right, plot of average DNA methylation (bisulfite-resistant cytosine $5 \mathrm{mC}+5 \mathrm{hmC})$ at the $\mathrm{DMR}^{48 \mathrm{~h} \text {-down }}(\mathrm{n}=1,097)$ in $48 \mathrm{~h}$-activated vs naïve $B$ cells. Average methylation is measured per $200 \mathrm{bp}$ bin.

(e) Heatmaps showing the kinetics of $5 \mathrm{hmC}$ at the $1,097 \mathrm{DMR}^{48 \mathrm{~h} \text {-down }}$ with decreased methylation $48 \mathrm{~h}$ postactivation. $5 \mathrm{hmC}$ enrichment is shown in normalized reads per $100 \mathrm{bp}$ bin.

(f) Venn diagram showing the overlap between regions with demethylation (DMR ${ }^{48 \mathrm{~h}-\text { down }}$ ) and hydroxymethylation $\left(\mathrm{DhmR}^{72 \mathrm{~h} \text {-up }}\right)$. Note that $49.3 \%(541 / 1097)$ of DMR are progressively hydroxymethylated post-activation.

(g) Strong enrichment for consensus binding motifs of NFKB, IRF:bZIP, bZIP, and other transcription factors in the 1,097 demethylating regions after $48 \mathrm{~h}$ activation $\left(D M R^{48 h-d o w n}\right)$. Random genomic regions were used as background for motif analysis. Y-axis indicates the fold enrichment versus background, circle size indicates the percentage of regions containing the respective motif, and the color indicates the significance $\left(\log _{10} p\right.$ vaule).

(h) $5 \mathrm{hmC}$ level tracks with enhancer activity. Left, differential active enhancers between naïve and 48h-activated $B$ cells were classified based on the significant difference in H3K27Ac and accessibility (ATAC-seq) into preferential 
bioRxiv preprint doi: https://doi.org/10.1101/438531; this version posted October 8,2018 . The copyright holder for this preprint (which was not certified by peer review) is the author/funder. All rights reserved. No reuse allowed without permission.

naïve-B-active enhancers (green, "Naïve>48h") and activated-B-active enhancers (orange, "48>naïve"). The remaining enhancers not meeting the above criteria were classified as common active enhancers (grey,

"Common"). Right, mean level of $5 \mathrm{hmC}$ per bin (50 bp) at the $+/-10 \mathrm{~kb}$ interval to the center was plotted for each type of active enhancers. Note that the $5 \mathrm{hmC}$ levels from the "Common" enhancers (middle) are also plotted as dotted lines for naïve-B-active (top) and activated-B-active enhancers (bottom) as reference.

Figure 2. Comparison of $5 \mathrm{hmC}$ modification in WT and Tet2/3 DKO B cells.

(a) Mean mRNA expression levels for TET family members (from RNA-seq) in WT naïve and activated B cells. TPM, transcript per million.

(b) Description of mice and flow chart of experiment.

(c) Tet2 and Tet 3 are efficiently deleted. Tet2 and Tet3 expression in B cells from tamoxifen-treated WT control and Tet2/3 DKO mice (described in Fig. 2b) were analyzed by qRT-PCR. Data were normalized to Gapdh within sample and subsequently to the value from WT. Representative of two independent experiments with three technical replicates is shown. ${ }^{* * *}, p<0.01$.

(d) Number of regions differentially marked with $5 \mathrm{hmC}(\mathrm{DhmR})$ between WT and Tet2/3 DKO B cells as a function of time after activation.

(e) Heatmaps showing the kinetics of $5 \mathrm{hmC}$ enrichment signals from WT (left panels) and Tet2/3-DKO (right panels) at the differentially hydroxymethylated regions $\left(\mathrm{DhmR}^{72 \mathrm{~h}}\right)$ between WT and DKO (72h, Fig. 2d). Regions with decreased $5 \mathrm{hmC}$ in DKO are shown on top $(\mathrm{WT}>\mathrm{DKO}, \mathrm{n}=2,139)$ and those with increased $5 \mathrm{hmC}$ on bottom (DKO>WT, $\mathrm{n}=184$ ). $5 \mathrm{hmC}$ enrichment is shown in normalized reads per $100 \mathrm{bp}$ bin.

(f) Strong enrichment for consensus RHD (NFKB), IRF:bZIP (IRF:BATF) and bZIP transcription factor binding motifs in the "TET-dependent" regions with decreased $5 \mathrm{hmC}$ in $72 \mathrm{~h}$-activated Tet2/3 DKO relative to WT B cells $\left(\mathrm{DhmR}^{72 \mathrm{~h}-\mathrm{WT}>\mathrm{DKO}}, \mathrm{n}=2,139\right)$. Common $5 \mathrm{hmC}$-enriched regions were used as background for analysis. $\mathrm{Y}$-axis indicates the fold enrichment versus background, circle size indicates the percentage of regions containing the respective motif, and the color indicates the significance $\left(\log _{10} p\right.$ vaule). See also Supplementary Fig. 2.

Figure S2, related to Fig. 2. Phenotypic features of WT and Tet2/3 DKO B cells.

(a) Comparable splenic mature B cell populations in Tet2/3-conditional deletion mice. WT (Tet2/3-flox Rosa26-LSLYFP) and DKO (Cre ${ }^{E R T 2}$ Tet2/3-flox Rosa26-LSL-YFP) mice were treated as in Fig. $\mathbf{2 b}$ and the phenotype of splenic $B$ cells was analyzed on day 7 after the initial tamoxifen injection. Plots were first gated on live single cells based on FSC/SSC (first panel) and total (WT) or YFP ${ }^{+}$(DKO) CD $19^{+} \mathrm{B}$ cells were subsequently gated (second panel), followed by the analysis of mature and immature B cells (third panel); and follicular (FO) and marginal zone (MZ) B cells (fourth panel).

(b) Total $5 \mathrm{hmC}$ levels in WT and Tet2/3 DKO B cells assessed by cytosine 5-methylenesulphonate (CMS) dot blot (see Materials and Methods). Note that $5 \mathrm{hmC}$ levels decrease in Tet2/3 DKO B cells only after several rounds of cell division (>48h).

(c) Histograms showing the distance from the TSS to the TET-regulated DhmR regions differentially marked with $5 \mathrm{hmC}$ in $72 \mathrm{~h}$-activated Tet2/3 DKO relative to WT B cells (see Fig. 2d, 2e). The 2,139 and 184 DhmRs with decreased (left, DhmR ${ }^{72 h}$ WT $>$ DKO) and increased (right, DhmR ${ }^{72 h}$ DKO $>$ WT) $5 \mathrm{hmC}$ after activated for $72 \mathrm{~h} 72 \mathrm{~h}-$ activated Tet2/3 DKO relative to WT B cells are located on average more than $10 \mathrm{~kb}$ from the closest TSS.

(d) Tet-mediated $5 \mathrm{hmC}$ modifications mark DNA demethylation. Left panels, heatmaps show the DNA modification status from naïve and 48h-activated WT B cells (WGBS, $5 \mathrm{mC}+5 \mathrm{hmC}$ ) at the 2,139 and $184 \mathrm{DhmR}$ regions with decreased (top, WT>DKO) and increased (bottom, DKO $>$ WT) $5 \mathrm{hmC}$ in $72 \mathrm{~h}$-activated Tet2/3 DKO vs WT B cells. Right panels, plots of the average decrease in bisulfite-resistant modifications $(5 \mathrm{mC}+5 \mathrm{hmC})$ per bin (200 bp) at these regions. The majority of the 2,139 WT>DKO DhmRs at $72 \mathrm{~h}$ show decreased modifications in activated WT B cells (top); the 184 DKO $>$ WT DhmR at $72 \mathrm{~h}$ are fully modified and unchanged in Tet2/3 DKO vs WT (bottom).

Figure 3. TET proteins facilitate class switch recombination (CSR) in vitro and in vivo.

(a) Flow chart of experiment to assess CSR in vivo. f.p., foot pad.

(b) Upper panels, flow cytometry plots showing equivalent frequencies of CD $19^{+} \mathrm{GL} 7^{+} \mathrm{Fas}{ }^{+}$germinal center B (GCB) cells at the draining popliteal lymph nodes from WT and Tet2/3 DKO mice after treated with tamoxifen and immunized with NP-OVA as indicated in (a). Lower panels, flow cytometry plots showing decreased frequencies of IgG1-switched cells among GCB cells in Tet2/3 DKO (YFP ${ }^{+}$GCB-gated) compared to WT mice (GCB-gated).

(c-f) Quantifications of experiments shown in (b). Data shown are aggregated results from two independent experiments. Means and standard errors are shown. WT, n=11; DKO, n=12. 
(g) Flow chart of experiment to assess CSR (IgG1 switching) in vitro. Cells were labeled with Cell-Trace violet and activated for 4 days with LPS $(25 \mathrm{ug} / \mathrm{mL})$ and $\mathrm{rmlL}-4(10 \mathrm{ng} / \mathrm{mL})$.

(h-i) Flow cytometry plots (h) and quantification of experiments (i) show decreased frequencies of lgG1-switched B cells in Tet2/3 DKO $(n=4)$ compared to WT $(n=4)$ mice. Data were representative from at least three independent experiments.

(j) Circular $\gamma 1$ transcript, generated after successful IgG1 switching, was quantified by qRT-PCR and normalized to Gapdh and then to the level of WT. Representative of two independent experiment is shown with three technical replicates.

(k) Flow chart of experiment to assess CSR (IgG1- and IgA-switching) in cell culture. Cells were activated for 5 days with anti-CD40 (1 ug/mL), rmIL-4 (10 ng/mL), rmlL-5 (10 ng/mL), and rhTGF $\beta(1 \mathrm{ng} / \mathrm{mL})$.

(I-m) Flow cytometry plots (I) and quantification of experiments $(m, n)$ showing decreased frequencies of $I g G 1-(m)$ and IgA-switched cells ( $\mathbf{n})$ in Tet2/3 DKO compared to WT cells. Data shown are representative from three independent experiments with three technical replicates. Statistical significance was calculated using unpaired two-tailed $t$-test. n.s., not significant. ${ }^{* * *}, p<0.01 .{ }^{*}, p<0.05$. See also Supplementary Fig. 3.

Figure S3, related to Fig. 3. TET family proteins are important for B-cell-intrinsic CSR.

(a) CSR defect is not caused by Cre activity. Cre ${ }^{E R T 2}$ Rosa26-LSL-YFP (Cre ${ }^{E R T 2}$ WT $)$ and $C r e^{E R T 2} \operatorname{Tet}^{f / / f l} T e t 3^{f / / f l}$ Rosa26-LSL-YFP (Cre ${ }^{E R T 2}$ DKO) mice were injected with tamoxifen as in Fig. $\mathbf{2 b}$. Isolated B cells were activated with LPS and IL-4 in the presence of 4-hydroxytamoxifen for 4 days and $\% \operatorname{lgG} 1^{+}$cells were analyzed (gated on live $\mathrm{CD} 19^{+} \mathrm{YFP}^{+}$). One experiment is shown. $\mathrm{n}=2$ for each genotype.

(b-c) (b) CSR defect is cell-intrinsic. (b) (Left) Tet2 ${ }^{+/+}$Tet $^{+/+}$WT CD45.1 and Tet2/3-DKO CD45.2 mice were treated as in Fig. $\mathbf{3 a}$ and isolated $B$ cells were labeled with Cell-Trace violet, 1:1 mixed, and activated with LPS and IL-4 for 4 days. (Right) Cells were gated based on CD45.1 and CD45.2 and the percentages of IgG1switched cells in WT and DKO are shown. Cells from the same well are connected with lines. (c) Co-cultured WT and Tet2/3-DKO B cells showed similar proliferation profiles. Data shown are representative of two independent experiments with four technical replicates for each genotype.

(d-f)(d) Flow chart of experiment to assess the importance of TET catalytic activity in CSR. (e) Flow cytometry plots and (f) quantification of WT and Tet2/3 DKO B cells transduced with empty vector (left panels), Tet2 wild-type catalytic domain (Tet2CD, middle panels), and Tet2 $\mathrm{HxD}$ mutant catalytic domain (Tet2CD ${ }^{\mathrm{HxD}}$, right panels) shows that TET catalytic activity can partly rescue the CSR to IgG1. Data shown are representative of two independent experiments with two technical replicates. n.s., not significant. ${ }^{* * *}, p<0.01 .{ }^{*}, p<0.05$.

(g-h) Deletion of all TET proteins Tet1/2/3 showed similar decrease in CSR compared to Tet2/3-DKO. (g) Tet1/2/3-flox Cre ${ }^{E R T 2}$ Rosa26-LSL-YFP (TKO) and control Tet1/2/3-flox Rosa26-LSL-YFP (WT) mice were treated with tamoxifen and immunized with NP-OVA as in Fig. 3a and GC response and CSR were analyzed on day 7. Flow cytometry plots showed the percentage of GCB $\left(C D 38^{\circ \mathrm{G}} \mathrm{GL} 7^{\mathrm{hi}}\right)$ in WT and Tet1/2/3-TKO lymph node cells gated on total (WT) and $\mathrm{YFP}^{+}$(TKO) live CD19 $\mathrm{B}$ cells (left panels). Antigen-specific (NP-PE) and classswitched cells (IgG1) were analyzed among the GCB. (h) Quantification of the experiments showed in (g). Data shown are aggregated results from two independent experiments. WT, $n=7 ; T K O, n=7$. Statistical significance was calculated using unpaired two-tailed $t$-test. n.s., not significant. ${ }^{* *}, p<0.01 .{ }^{*}, p<0.05$.

Figure 4. Tet2/3 facilitate CSR by regulating expression of the cytidine deaminase AID.

(a-b) Decreased expression of AID mRNA and proteins in Tet2/3-DKO. (a) qRT-PCR analysis Aicda mRNA expression in WT and Tet2/3-DKO B cells activated 4 days with LPS and IL4. Aicda expression was normalized to Gapdh and then to the level in WT. Result shows $\sim 50 \%$ decrease of Aicda mRNA expression in Tet2/3 DKO relative to WT B cells. Data shown are representative of two independent experiments with three technical replicates. ${ }^{*}, p<0.05$. For results of genome-wide RNA sequencing, see Supplementary Fig. 4.

(b) Immunoblotting of whole cell lysates showed a substantial decrease of AID and Tet2 protein expression in Tet2/3-DKO relative to WT B cells activated for 4 days. Left lane contains lysate from the AID-KO CH12 B cells as a control for the specificity of anti-AID antibody. $\beta$-Actin was used as loading control. Data shown are representative of two independent experiments.

(c-d) Expression of catalytically active AID restores CSR in Tet2/3-DKO. WT and Tet2/3-DKO B cells were retrovirally transduced with empty vector expressing Thy1.1 (left panels), wild-type AID (AID ${ }^{\text {WT }}$, middle panels), or catalytically inactive AID (AID ${ }^{\mathrm{MUT}}$, right panels). Cells were gated on live transduced B cells (CD19 $9^{+}$Thy $1.1^{+}$). Representative flow cytometry plots (c) and quantification (d) are shown. Data shown are representative of three independent experiments. n.s., not significant. ${ }^{* *}, p<0.01$. See also Supplementary Fig. 4. 
Figure S4, related to Fig. 4. Decreased Aicda expression in Tet2/3-DKO B cells.

WT and Tet2/3-DKO B cells were activated as in Fig. $\mathbf{3 g}$ and the transcriptomes were analyzed by RNA-seq (see Materials and Methods and Supplementary Table S1 for details).

(a) Number of differentially expressed genes between WT and Tet2/3-DKO B cells as a function of time after activation. Relatively few genes show alterations in their expression.

(b) List of all differentially expressed genes between WT and Tet2/3-DKO B cells. Aicda (indicated by red arrows) was one of the genes expressed significantly lower in DKO. Color scale indicates $\log _{2}$ fold change between WT and DKO.

(c) Tet2 and Tet3 are required for potent Aicda expression. Aicda mRNA expression was analyzed by qRT-PCR as in Fig. $4 \mathrm{a}$ as a function of time after activation. Results showed a consistent $50 \%$ decrease in Tet $2 / 3$ DKO relative to WT B cells.

(d-i) Haploinsufficiency of Aicda results in decreased CSR. Mice with indicated genotypes were immunized with 10ug of NP-OVA mixed with Alum via footpad and the draining lymph nodes were analyzed by FACS day 7 postimmunization. Heterozygous Aicda-Cre mice were used to model Aicda haploinsufficiency as the knocked-in Cre recombinase disrupted the endogenous Aicda expression. Representative FACS analysis of (d) germinal center B cells $\left(\mathrm{GCB} ; \mathrm{CD} 38^{\mathrm{lo}} \mathrm{GL} 7^{\mathrm{hi}}\right)$ and $(\mathrm{e}) \mathrm{CSR}$ to $\operatorname{lgG} 1$. (f-i) Statistical analyses of the populations are shown $(\mathrm{n}=4$ each). Data shown are representative of two independent experiments. Means and standard errors are shown. Unpaired two-tailed $t$-test was used to calculate statistical significance and the $p$ values are indicated.

(j) Tet2 and Tet3 are not required for expression of germline transcripts. WT and DKO B cells were activated for 4 days, and $\mu$ and $\gamma 1$ germline transcripts were analyzed by qRT-PCR. Data were normalized to Gapdh and to WT level as in Fig. 4a. n.s., not significant.

Figure 5. Tet2 and Tet3 control Aicda expression via TET-responsive elements TetE1 and TetE2.

Diagram shows two conserved TET-responsive elements TetE1 and TetE2 at the 5' of the Aicda gene (labeled with green rectangles and grey shades).

(a) Top two tracks. ChIP-seq analysis showed that Tet2 (blue track) specifically bound to multiple elements in the Aicda locus (mm10; chr6:122,523,500-122,576,500) after activation when compared to Ig control (grey track). Middle tracks (green). Increased H3K27 acetylation at the upstream and intronic regulatory elements of Aicda after activation. Bottom tracks. Activation induced DNA demethylation at TetE1 and TetE2. Whole genome bisulfite sequencing (WGBS) showing DNA methylation $(5 \mathrm{mC}+5 \mathrm{hmC})$ in naïve and $48 \mathrm{~h}$-activated B cells ( $m C G$, black tracks). CpGs included in the analysis are indicated by red lines (red track). Bottom track indicates the conserved DNA elements among placental animals ("Conserve"). Previously identified super-enhancer is indicated. For Tet2 and Ig, scales indicate per 10 millions reads; for H3K27Ac, quantile-normalized reads; for bisulfite sequencing, percentage of bisulfite-resistant cytosine.

(b) Activation induced Tet2/3-dependent $5 \mathrm{hmC}$ deposition at Aicda distal elements. WT and Tet2/3-DKO B cells were activated as in Fig. $\mathbf{3 g}$ with LPS and IL-4 as a function of time. DNA was purified and $5 \mathrm{hmC}$ enrichment was detected by CMS-IP (see Materials and Methods). Significant differential $5 \mathrm{hmC}$-enriched regions between WT and DKO after $72 \mathrm{~h}$-activation were indicated at the bottom (WT>DKO DhmR $\left.{ }^{72 \mathrm{~h}}\right)$. Scales indicate quantile-normalized reads.

(c) Tet2/3 deposit 5hmC and demethylate Aicda TET-responsive element TetE1 and promoter. CpG modifications ( $5 \mathrm{hmC}, 5 \mathrm{mC}$, and C) at TetE1 (top panels) and promoter (bottom panels) were analyzed by oxidative bisulfite sequencing (oxBS-seq; Materials and Methods) using DNA isolated from WT and Tet2/3-DKO $B$ cells before and after activation. Although $5 \mathrm{hmC}$ and $5 \mathrm{mC}$ can be distinguished by oxBS-seq, unmodified $\mathrm{C}$ and minuscule amount of $\mathrm{fC}$ and $\mathrm{caC}$ were recognized as " $\mathrm{C}$ ", all of which are sensitive to deamination by bisulfite treatment. See also Supplementary Fig. 5.

Figure S5, related to Fig. 5. The TET-responsive element TetE1 regulates CSR and Aicda mRNA expression in the $\mathrm{CH} 12 \mathrm{~B}$ cell line.

(a) Diagram depicts the relative position of TET-responsive elements TetE1 and TetE2 to previously identified Aicda distal and intronic enhancers. "Region" IV, II, III are from Tran et al., 2010; "CNS" V-X are from Crouch et al., 2007; "Enhancer" E1-E5 from Kieffer-Kwon et al., 2013. Note that the promoter-proximal element is not depicted. Coordinate for the shown locus is chr6:122,523,500-122,576,500 (mm10).

(b-e) TetE1 is important for regulating Aicda expression and CSR. (b) Scheme for TetE1 deletion in $\mathrm{CH} 12$ cells with CRISPR. (c) Four clones were identified with homozygous deletion of TetE1 as examined by PCR followed by gel electrophoresis. A clone with heterozygous deletion (Het) and a WT control were showed as controls. (d-e) WT and TetE1-deletion clones were stimulated with CIT (anti-CD40, IL-4, TGF $\beta$ ) for two days. (d) Aicda mRNA 
bioRxiv preprint doi: https://doi.org/10.1101/438531; this version posted October 8,2018 . The copyright holder for this preprint (which was not certified by peer review) is the author/funder. All rights reserved. No reuse allowed without permission.

expression and (e) CSR to IgA was analyzed by qRT-PCR and flow cytometry, respectively. Results showed that deletion of TetE1 decreased both Aicda mRNA expression and abrogated CSR.

Figure 6. Tet proteins sustain enhancer accessibility.

(a) B cell activation induced global accessibility changes. WT B cells were activated with LPS and IL-4 as a function of time and the chromatin accessibility was profiled by ATAC-seq. Numbers are shown for the differential accessible regions (DARs) between activated (act.) and naïve B cells with FDR $<0.05$ and fold change above $\log _{2}(1.5)$ or below $\log _{2}(0.67)$.

(b) Loss of TET proteins decreased accessibility at later time point. Numbers of DARs between WT and Tet2/3-DKO B cells activated across time points are shown. Note that the difference between WT and DKO was minimal at time points earlier than $72 \mathrm{~h}$. DARs were selected based on FDR $<0.05$ and fold change above $\log _{2}(1.5)$ or below $\log _{2}(0.67)$

(c) Tet2/3-dependent accessible regions are hydroxymethylated. Heatmaps show the kinetics of $5 \mathrm{hmC}$ modification at the differential accessible regions (DARs) between WT and Tet2/3-DKO. Regions that are more accessible in WT (WT>DKO, $n=292$ ), less accessible in WT (DKO>WT, n=129), and commonly accessible $(n=27,716)$ are shown on top, middle, and bottom panels, respectively. Note that the WT>DKO DARs show progressive $5 \mathrm{hmC}$ enrichment only in WT (top left panels) but not in DKO (top right panels), demonstrating the $5 \mathrm{hmC}$ modification at these regions is Tet2/3-dependent. The DKO>WT DARs $(n=129)$ and common regions $(n=27716)$ show no apparent difference between naïve and activated $B$ cells and between $5 \mathrm{hmC}$ from WT and DKO B cells. Note that the length of the commonly accessible regions is not to scale compared to DARs. $5 \mathrm{hmC}$ enrichment is shown as normalized reads per $100 \mathrm{bp}$ bin.

(d) Tet2 and Tet3 maintain chromatin accessibility at Aicda Tet-responsive elements TetE1 and TetE2. Genome browser view of ATAC-seq data showing the accessibility profile at Aicda locus in WT (blue, top 4 tracks) and DKO B cells (red, bottom 4 tracks). Note that TetE1 and TetE2 were among the DAR at $72 \mathrm{~h}$ (DAR ${ }^{72 \mathrm{~h}}$ WT>DKO) as indicated at the bottom. Coordinate for locus is chr6:122,523,500-122,576,500 (mm10).

(e) Plot of mean chromatin accessibility at the DARs between WT and Tet2/3-DKO B cells after 72h-activation (as in (c) top and middle panels). Top panels, WT>DKO DARs $(n=292)$; bottom panels, DKO>WT DARs $(n=129)$. Yaxes indicate the mean ATAC signals (normalized ATAC-seq reads per $100 \mathrm{bp}$ bin) from WT (blue line) and DKO (red line) B cells activated as indicated. Note that the difference between WT and DKO is apparent at $72 \mathrm{~h}$. See also Supplementary Fig. 6.

\section{Supplementary Figure 6, related to Figure 6. Analysis of Tet-dependent accessible regions.}

(a) Correlation between $5 \mathrm{hmC}$ and accessibility. At the differentially $5 \mathrm{hmC}$-enriched regions between WT and DKO (DhmR ${ }^{72 h-u p}, n=8454$, Fig. 1c), mean chromatin accessibility detected by ATAC-seq was shown for each region. Note that the closely correlated increase of both $5 \mathrm{hmC}$ and accessibility (blue, ATAC-WT). Loss of Tet $2 / 3$ resulted in an observed decrease in accessibility, corresponding to the results from Fig. 6c. Statistical significance between WT and DKO at each time point was calculated by Kolmogorov-Smirnov test with Bonferroni correction using the family-wise error rate. n.s., not significant. ${ }^{* * *}, p$ adj. $<0.01$. The exact adjusted $p$ values are $0.06,6.01 \mathrm{e}-05,1.79 \mathrm{e}-07,3.611 \mathrm{e}-11$ for $0 \mathrm{~h}, 24 \mathrm{~h}, 48 \mathrm{~h}, 72 \mathrm{~h}$, respectively.

(b)Tet-facilitated accessibility at distal elements. Histograms showing the distance of DARs (WT>DKO and $\mathrm{DKO}>\mathrm{WT}$ ) and commonly accessible regions (Common) from the closest TSS. Majority of the Tet-facilitated accessible regions (WT>DKO, $n=292$ ) are distal elements (>1000bp; 92.8\%).

(c)Tet2/3-dependent accessible regions are hydroxymethylated. Line plots showing the kinetics of mean $5 \mathrm{hmC}$ modification at the differential accessible regions (DARs) between WT and Tet2/3-DKO for the data depicted in Fig. 6c. Regions that are more accessible in WT (WT>DKO, $n=292$ ), less accessible in WT (DKO>WT, $n=129)$, and commonly accessible $(n=27,716)$ are shown on top, middle, and bottom panels, respectively. $5 \mathrm{hmC}$ enrichment is shown as normalized reads per $100 \mathrm{bp}$ bin. Note the Tet-dependent $5 \mathrm{hmC}$ modification at these DARs (compare $72 h$ panels).

(d)Tet-facilitated accessible regions further demethylated after activation. Left, heatmaps showing the DNA modifications status $(5 \mathrm{mC}+5 \mathrm{hmC})$ in naïve and $48 \mathrm{~h}$-stimualted WT B cells at the WT>DKO DARs or Tetfacilitated accessible regions $(n=292)$ and DKO>WT DARs $(n=129)$. Right, plots summarized the results for the heatmaps (left) with $y$-axis indicates the level of bisulfite-resistant cytosine $(5 \mathrm{mC}+5 \mathrm{hmC})$. In WT B cells, regions that lose accessibility in Tet2/3 DKO B cells relative to WT (WT>DKO) also show a decrease in modifications (mostly $5 \mathrm{mC}$ ) after activation.

(e) Enrichment for consensus IRF:bZIP (IRF:BATF) and bZIP transcription factor binding motifs in the 292 Tetfacilitated accessible regions, which shows increased accessibility in WT relative to Tet2/3 DKO B cells at $72 \mathrm{~h}$. 
bioRxiv preprint doi: https://doi.org/10.1101/438531; this version posted October 8,2018 . The copyright holder for this preprint (which was not certified by peer review) is the author/funder. All rights reserved. No reuse allowed without permission.

No significant motif enrichment was detected at the DKO>WT DAR $(n=129)$. Commonly accessible regions were used as background for the analysis. Y-axis indicates the fold enrichment versus background, circle size indicates the percentage of regions containing the respective motif, and the color indicates the significance $\left(\log _{10}\right.$ $p$ vaule).

(f) B cell activation induces recruitment of chromatin regulators to Aicda distal elements. Genome browser view of ChIP-seq data showing $B$ cell activation induces the binding of the chromatin remodelling complex components Brg1 and Chd4, and histone acetyltransferases p300 and Gcn5 to the TET-responsive Aicda elements TetE1 and TetE2 in naïve and activated WT B cells. Scale indicates reads per 10 millions. Coordinate for locus is chr6:122,523,500-122,576,500 (mm10).

Figure 7. BATF recruits Tet proteins to hydroxymethylated Aicda Tet-responsive element TetE1.

(a) Mean mRNA expression level of Batf family members (Batf1-3) in B cells activated with LPS and IL-4 as a function of time. Data shown are from RNA-seq with two independent replicates. TPM, transcript per million.

(b-c) BATF binding correlates with $5 \mathrm{hmC}$-enrichment. WT BATF peaks $(\mathrm{n}=11,640)$ were divided into two clusters based on the pattern of $5 \mathrm{hmC}$ distribution. (b) Cluster 1 ( $\mathrm{n}=2,198$; top panels) showed a broad $5 \mathrm{hmC}$ distribution, with the $5 \mathrm{hmC}$ level remained unchanged after activation and in the absence of Tet2/3 (upper panels, compare " $5 \mathrm{hmC}$ from WT" to " $5 \mathrm{hmC}$ from DKO"). In contrast, a substantial portion of regions in cluster 2 $(n=9,422)$ showed a progressive Tet-dependent $5 \mathrm{hmC}$ modification after activation (lower panels) and is further illustrated in (c) as line plots. Data shown are mean enrichment per $100 \mathrm{bp}$ bin.

(d) Recruitment of BATF and other transcription factors to Aicda enhancers. Upper two tracks, genome browser view of BATF-binding in 72h-activated WT (blue) and Tet2/3-DKO B cells (red) at the Aicda locus. Note that the major BATF-binding sites are at TetE1 and TetE2, and the loss of Tet2/3 has no significant effect on BATF recruitment (compare WT and DKO; also see Supplementary Fig. 7f; two independent experiments). Activation also induced E2A and PU.1 binding to Aicda enhancers (orange and purple tracks). Coordinate for locus is chr6:122,523,500-122,576,500 (mm10). See also Supplementary Fig. 7.

(e) BATF is required for $5 \mathrm{hmC}$ modification at TetE1. B cells were isolated from WT and Batf-KO and activated with LPS and IL-4 for 4 days. $5 \mathrm{hmC}$ modification at TetE1 was quantified using AbaSI-qPCR (see Materials and Methods).

Figure S7, related to Fig. 7. AP-1 proteins in activated B cells.

(a) Analysis of temporal gene expression modules. Gene expression in WT cells activated for various times were analyzed and genes were clustered based on the temporal expression patterns. Six clusters (C1-C6), or expression patterns, were identified. Aicda and Batf are found in C2 and C4, respectively. Y-axis indicates the Zscore calculated using RPKM. X-axis indicates the time post-activation. For details, see Materials and Methods and Supplementary Table S2.

(b-d) Expression of AP-1 proteins. Mean mRNA expression of selected AP-1 proteins in naïve and activated B cells including Fos family (b), Jun family (c), and Bach family (d). Note that LPS/IL-4 simulation decreased the expression of Fos, Fosb, Jun, and JunB followed by a gradual increase in JunB expression (b, c). The high basal expression of these factors could potentially attribute to minor B cell subset, including memory B cells. (d) Expression of Bach2, a transcription factor expressed in naïve and memory cells, also decreased with a delayed kinetics at $72 \mathrm{~h}$ post-activation. Data shown are from RNA-seq with two independent replicates. TPM, transcript per million.

(e) Batf is required for CSR. B cells were isolated from WT and Batf-KO and activated with LPS and IL-4 for 4 days. Class switch recombination to IgG1 was analyzed by flow cytometry (gated on live CD19 ${ }^{+}$). Data shown is representative of two independent experiments ( $n=4$ each). Means and standard errors are shown. Statistical significance was calculated using unpaired two-tailed $t$-test. ${ }^{* *}, p<0.01$.

(f) Tet proteins are not necessary for genome-wide BATF binding. Plots show the highly similar distribution of BATF in WT and DKO 72h-activated B cells as analyzed by ChIP-seq with two independent replicates. Data shown are the comparison of the BATF enrichment in WT and DKO at the 26,909 regions integrated from the joined peaks from two replicates each of WT and DKO. Axes depict the $\log _{2}$ rpkm (read per kilo base per million) using quantile-normalized reads for each regions analyzed. Note that no region was significantly differenct between WT and DKO using an adjusted $p$ value at 0.05 .

(g-h) Expression of E-box and Ets family proteins. Mean mRNA expression for E-box and Ets family proteins are shown. Data shown are from RNA-seq with two independent replicates. TPM, transcript per million.

(i) JUNB and BATF bind to AICDA enhancers in human B cells. JUNB and BATF bindings in human B cell lymphoblast GM12878 at the AICDA locus are shown (Hg38; chr12:8,598,356-8,655,770). The approximate 
bioRxiv preprint doi: https://doi.org/10.1101/438531; this version posted October 8,2018 . The copyright holder for this preprint (which was not certified by peer review) is the author/funder. All rights reserved. No reuse allowed without permission.

locations for TETE1 and TETE2 are indicated. Data were originally from ENCODE project, processed by CistromeDB, and were viewed using WashU Epigenome Browser. 


\section{Materials and Methods}

Mice. Tet $2^{f / f l}$ and Tet $3^{f / f l}$ mice were generated as previously described ${ }^{55,56}$. C57BL/6J (000664), Ubc-CreERT2 (008085; described as Cre ${ }^{\text {ERT2 }}$ herein), Rosa26-LSL-EYFP (006148), and AID-Cre (007770) were obtained form Jackson Laboratory. All mice used were 8-16 weeks in the C57BL/6 background and kept in specific-pathogen free animal facilitate at La Jolla Institute and were used according to protocols approved by the Institutional Animal Care and Use Committee. To induce Cre ${ }^{\text {ERT2 }}$-mediated deletion, Cre-expressing and control mice were intraperitoneally injected with $2 \mathrm{mg}$ tamoxifen (Sigma) dissolved in $100 \mathrm{uL}$ corn oil (Sigma) daily for 5 days.

Immunization. For 4-hydroxy-3-nitrophenylacetyl-conjugated ovalbumin (NP-OVA; Biosearch) immunization, the hapten-conjugated protein was diluted to $1 \mathrm{mg} / \mathrm{mL}$ in PBS was mixed with 1 volume of Alhydrogel (Invivogen) and injected into hind footpads (10 ug in $20 \mathrm{uL}$ per injection). Germinal center response was analyzed 7 days later and the two draining popliteal lymph nodes were pooled for analysis. Hapten-specific B cells were identified by positive staining with NP-phycoerythrin (BioSearch Technologies).

B cell isolation and class switch recombination (CSR). B cells were isolated with EasySep Mouse B cell isolation kit (Stem Cell Technology, Canada) from splenocytes. To induce class switch recombination from IgM to IgG1, B cells $\left(5 \times 10^{5}-1 \times 10^{6}\right.$ cells $\left./ \mathrm{mL}\right)$ were activated with $25 \mathrm{ug} / \mathrm{mL}$ LPS from E. coli O55:B5 (Sigma, St. Louis, MO) and $10 \mathrm{ng} / \mathrm{mL} \mathrm{rmL-} 4$ at $37^{\circ} \mathrm{C} 5 \% \mathrm{CO}_{2}$. For IgA switching, cells were activated with anti-CD40 (1 ug/mL, clone 1C10, Biolegend), rmlL-4 (10 ng/mL, Peprotech), rmlL-5 (10 g/mL, Peprotech), and rhTGF $\beta 1$ (1 ng/mL). Media were composed of RPMI1640 (Thermo Fisher, Waltham, MA) supplemented with 10\% FBS, 1x MEM non-essential amino acids, $10 \mathrm{mM}$ HEPES, $2 \mathrm{mM}$ Glutamax, $1 \mathrm{mM}$ sodium pyruvate, $55 \mathrm{uM}$ 2-mercaptoethanol, and $50 \mathrm{ug} / \mathrm{mL}$ gentamicin (all from Thermo Fisher, Waltham, MA). To enhance Cre ${ }^{E R T 2}$-mediated deletion, cells from Cre ${ }^{E R T 2}$ mice were cultured in the presence of 1 uM 4-hydroxytamoxifen (Tocris). All cytokines used above were from Peprotech (Rocky Hill, NJ).

Retroviral transduction and two-step CSR. Retrovirus was produced by transfecting PlatE cells with MSCVbased retroviral vectors and pCL-Eco. Naïve B cells were stimulated with $5 \mathrm{ug} / \mathrm{mL} F\left(a b^{\prime}\right)_{2}$ goat anti-mouse IgM (Jackson Immuno Research) and $10 \mathrm{ug} / \mathrm{mL}$ LPS at $1 \times 10^{6}$ cells $/ \mathrm{mL}$ for $24-48$ hours. Retrovirus was added to the cells in the presence of $20 \mathrm{mM}$ HEPES and $0.8 \mathrm{ug} / \mathrm{mL}$ Polybrene (Millipore) and centrifuged at $3,000 \mathrm{rpm}$ at $32^{\circ} \mathrm{C}$ for 90 mins. Cells were transferred back to $37^{\circ} \mathrm{C} 5 \% \mathrm{CO}_{2}$ incubator for another 24 hours. To induce CSR, cells were washed once with warm media and activated with LPS and IL-4 as above for 48 hours. Under this condition, CSR was inhibited and started to class switch only after LPS/IL-4 activation.

Flow cytometry. Primary cells and in vitro cultured cells were stained in FACS buffer (1\% bovine serum albumin, $1 \mathrm{mM}$ EDTA, and $0.05 \%$ sodium azide in PBS) with indicated antibodies for 30 mins on ice. Cells were washed and then fixed with 1\% paraformaldehyde (diluted from 4\% with PBS; Affymetrix) before FACS analysis using FACS Canto II and FACS LSR II (BD Biosciences). Antibodies and dye were from BioLegend, eBioscience, and BD Pharmingen. Data were analyzed with FlowJo (FlowJo LLC, Ashland, OR).

Immunoblotting. Proteins isolated from B cells with RIPA buffer were resolved using NuPAGE 4-12\% Bis-Tris gel (ThermoFisher) and transferred from gel to PVDF membrane using Wet/Tank Blotting Systems (Bio-Rad). Membrane was blocked with 5\% non-fat milk (Bob's red mill) in TBSTE buffer (50 mM Tris- $\mathrm{HCl} \mathrm{pH} 7.4,150 \mathrm{mM}$ $\mathrm{NaCl}, 0.05 \%$ Tween-20, $1 \mathrm{mM}$ EDTA), incubated with indicated primary antibodies, followed by secondary antibodies conjugated with horseradish peroxidase (HRP) and signal was detected with enhance chemiluminescence reagents and X-ray film.

RNA extraction, cDNA synthesis, and quantitative RT-PCR. Total RNA was isolated with RNeasy plus kit (Qiagen, Germnay) or with Trizol (ThermoFisher, Waltham, MA) following manufactures' instruction. cDNA was synthesized using SuperScript III reverse transcriptase (ThermoFisher) and quantitative RT-PCR was performed 
using FastStart Universal SYBR Green Master mix (Roche, Germany) on a StepOnePlus real-time PCR system (ThermoFisher). Gene expression was normalized to Gapdh. Primer are listed in Supplementary Table 3.

\section{Bisulfite- (BS) and oxidative-bisulfite- (oxBS) sequencing}

The BS and oxBS procedures were performed as previously described ${ }^{28}$. Briefly, three PCR products containing C, $\mathrm{mC}$, or $\mathrm{hmC}$ pertaining to different regions of $\Lambda$ phage genome were used as spike-ins at a ratio of 1:200 of the genomic DNA. $1.5 \mu \mathrm{g}$ of genomic DNA mixed with spike-ins was ethanol precipitated of which $1 \mu \mathrm{g}$ of the DNA was oxidized with potassium perruthenate $\left(\mathrm{KRuO}_{4}\right.$; Sigma) prior to bisulfite (BS) treatment (for oxBS) using MethylCode bisulfite conversion kit (ThermoFisher) and $0.5 \mu \mathrm{g}$ of DNA was directly used for BS treatment. The BS and oxBS treated DNA were amplified using respective PCR primers and as well as primers specific to the spike-in PCR products with KAPA Uracil ${ }^{+}$PCR mix (Roche). The amplified products were pooled and libraries were prepared using the NEB Ultra II library preparation kit (NEB) according the manufacturer. The libraries were sequenced paired-end $250 \mathrm{bp}$ by $250 \mathrm{bp}$ using MiSeq with the MiSeq reagent kit v2 (500-cycles; Illumina). Primer are listed in Supplementary Table 3.

Genome-wide $5 \mathrm{hmC}$ mapping by cytosine-5-methylenesulfonate immunoprecipitation (CMS-IP). CMS-IP was performed similar to perviously described ${ }^{4,24,25}$. Briefly, genomic DNA isolated from naïve and activated $B$ cells was spiked with unmethylated lambda phage cl857 Sam7 DNA (Promega, Madison, WI) and a PCR amplicon from puromycin resistant gene at a ratio of 200:1 and 100,000:1, respectively. DNA (5-10 ug in 130 uL TE buffer) was sheared with a Covaris E220 (Covaris) using microTUBE for 4 mins. DNA was cleaned-up with AmureXP beads, processed with NEBNext End Repair and A-tail Modules (NEB, Ipswich, MA), and ligated to methylated Illumina adaptors (NEB). DNA was then bisulfite-treated (MethylCode, ThermoFisher), denatured, and immunoprecipitated with anti-CMS serum (in house) and mixture of protein $A$ and $G$ dynabeads (ThermoFisher). Libraries for immunoprecipitated DNA were generated by PCR with barcoded primers (NEBNext Multiplex Oligos for Illumina, NEB) for 15 cycles using KAPA HiFi HotStart Uracil+ ReadyMix (Roche), followed by a cleanup with AmpureXP beads (Beckman Coulter), and sequenced with a HiSeq2500 (Illuminia, San Diego, CA) with paired-end 50 bp reads.

Locus specific analysis of $5 \mathrm{hmC}$ with AbaSI-qPCR. Genomic DNA (200 $\mathrm{ng}$ ) was treated with T4 betaglucosyltransferase (ThermoFisher) in the presence of UDP-glucose to glycosylate $5 \mathrm{hmC}$ at $37^{\circ} \mathrm{C}$ overnight. Half of the reaction was digested with AbaSI (NEB), which is specifically active for glycosylated $5 \mathrm{hmC}$, for 4 hours at $25^{\circ} \mathrm{C}$ followed by 15 mins at $65^{\circ} \mathrm{C}$ to inactivate enzymes. The uncut sample was processed as above without the addition of AbaSI. Equal amount of DNA from the above reactions was used as template for real-time PCR as described for RNA qRT-PCR using primers TetE1-CMS-qF and TetE1-CMS-qR. To monitor the degree of digestion, samples were spiked-in 1 pg control DNA with a single 5hmC-modified CpG (EpiMark 5-hmC and 5-mC Analysis Kit; NEB). The relative amount of $5 \mathrm{hmC}$ was calculated by the percentage of decrease in qPCR signals in digested half relative to undigested half. As control to monitor non-specific digestion (not show), a genomic region containing CpG motifs but without $5 \mathrm{hmC}$ modification in B cells (Foxp3 CNS2) was used as a control with Foxp3-CNS2-qF and Foxp3CNS2-qR. Primers are listed in Supplementary Table 3.

DNA dot blot. DNA dot blot was performed as previously described ${ }^{4,28}$. To analyze $5 \mathrm{hmC}$ abundance, genomic DNA was treated with sodium bisulfite as above. DNA was diluted two-fold serially with TE buffer, denatured in 0.4 $\mathrm{M}$ sodium hydroxide and $10 \mathrm{mM}$ EDTA at $95^{\circ} \mathrm{C}$ for $10 \mathrm{mins}$, and then immediately chilled on ice. Equally volume of ice-cold $2 \mathrm{M}$ ammonium acetate $\mathrm{pH} 7.0$ was added and incubated on ice for 10 mins. Denatured DNA were spotted on a nitrocellulose membrane using a Bio-Dot apparatus (Bio-Rad), washed with 2x SSC buffer (300 mM NaCl and $30 \mathrm{mM}$ sodium citrate), and baked in a vacuum oven at $80^{\circ} \mathrm{C}$ for 2 hours. To detect CMS, membrane was rehydrated with TBSTE buffer and blocked with $5 \%$ non-fat milk (Bob's red mill) in TBSTE buffer. CMS was detected with primary rabbit anti-CMS antisera (in house) following the procedures above for Immunoblotting. 
Chromatin Immunoprecipitation sequencing (ChIP-seq). Chromatin immunoprecipitation was performed as described before ${ }^{4}$. Briefly, cells were fixed with $1 \%$ formaldehyde (ThermoFisher) at room temperature for 10 mins at $1 \times 10^{6} \mathrm{cell} / \mathrm{mL}$ in media, quenched with $125 \mathrm{mM}$ glycine, washed twice with ice cold PBS. Cells were pelleted, snap-froze with liquid nitrogen, and store at $-80^{\circ} \mathrm{C}$ until use. For Tet2-ChIP, activated cells were centrifugated at 250 $\mathrm{x} g$ for 5 mins and cell pellets were resuspended in $37^{\circ} \mathrm{C}$ PBS with $2 \mathrm{mM}$ disuccinimidyl glutarate to crosslink proteins for 30 mins at room temperature. Formaldehyde was added to a final concentration of $1 \%$ and the cells were incubated at room temperature for 10 mins with nutation. Quenching and cell storage were performed as above. To isolate nuclei for sonication, cell pellets were thawed on ice and lysed with lysis buffer (50 mM HEPES pH $7.5,140 \mathrm{mM} \mathrm{NaCl}, 1 \mathrm{mM}$ EDTA, $10 \%$ glycerol, $0.5 \% \mathrm{NP} 40,0.25 \%$ Triton-X100) for 10 mins at $4^{\circ} \mathrm{C}$ with rotation, washed once with washing buffer (10 mM Tris- $\mathrm{HCl} \mathrm{pH} 8.0,200 \mathrm{mM} \mathrm{NaCl}, 1 \mathrm{mM}$ EDTA, $0.5 \mathrm{mM}$ EGTA) and twice with shearing buffer (10 mM Tris- $\mathrm{HCl} \mathrm{pH} 8.0,1 \mathrm{mM}$ EDTA, $0.1 \%$ SDS). Nuclei were resuspended in $1 \mathrm{~mL}$ shearing buffer and sonicated with Covaris E220 using $1 \mathrm{~mL}$ milliTUBE (Covaris, Woburn, MA) for 18-20 minutes (Duty Cycle $5 \%$, intensity 140 Watts, cycles per burst 200). After sonication, insoluble debris was removed by centrifugation at $20,000 \times \mathrm{g}$. Buffer for chromatin was adjusted with 1 volume of $2 \times$ conversion buffer $(10 \mathrm{mM} \mathrm{Tris-} \mathrm{HCl} \mathrm{pH} 7.5,280$ $\mathrm{mM} \mathrm{NaCl}, 1 \mathrm{mM}$ EDTA, $1 \mathrm{mM}$ EGTA, $0.2 \%$ sodium deoxycholate, $0.2 \%$ Triton- $\mathrm{X} 100,1 \%$ Halt protease inhibitors with (for H3K27Ac) or without (for BATF, Tet2) $0.1 \%$ SDS. Chromatin was pre-cleared with washed protein A dynabeads (ThermoFisher) for 2 hours, incubated with antibodies and protein $A$ dynabeads overnight (all procedures were at $4^{\circ} \mathrm{C}$ with rotation). For H3K27Ac ChIP, bead-bound chromatin was washed twice with RIPA buffer $(50 \mathrm{mM} \mathrm{Tris-HCl}$ $\mathrm{pH}$ 8.0, $150 \mathrm{mM} \mathrm{NaCl}, 1 \mathrm{mM}$ EDTA, $0.5 \%$ sodium deoxycholate, $1 \% \mathrm{NP}-40,0.1 \% \mathrm{SDS}$ ), once with high salt wash buffer (50 mM Tris-HCl pH 8.0, $500 \mathrm{mM} \mathrm{NaCl}, 1 \mathrm{mM}$ EDTA, 1\% NP-40, 0.1\% SDS), and once with TE (10 mM Tris$\mathrm{HCl} \mathrm{pH} \mathrm{8.0,} 1 \mathrm{mM}$ EDTA). For BATF ChIP, all wash buffers were as above but without SDS. For Tet2 ChIP, beads were washed three times with RIPA buffer without SDS and once with TE. Chromatin was eluted from beads with elution buffer ( $100 \mathrm{mM} \mathrm{NaHCO}_{3}, 1 \%$ SDS, $1 \mathrm{mg} / \mathrm{mL}$ RNaseA; Qiagen) twice for 30 mins each at $37^{\circ} \mathrm{C}$ with constant shaking. $\mathrm{NaCl}$ and proteinase $\mathrm{K}$ (Ambion) were added to the eluted chromatin at concentrations of $250 \mathrm{mM}$ and 0.5 $\mathrm{mg} / \mathrm{mL}$, respectively, and de-crosslinked at $65^{\circ} \mathrm{C}$ overnight with constant shaking. DNA was purified with Zymo ChIP DNA Clean \& Concentrator-Capped Column (Zymo Research, Irvine, CA). Library was prepared with NEB Ultra II library prep kit (NEB) following manufacture's instruction and was sequenced on an Illumina Hiseq 2500 (single-end 50 bp reads).

ATAC-seq. Procedures were as described ${ }^{4}$. Briefly, 50,000 cells were collected by centrifugation and washed once with 50uL ice-cold PBS and centrifuged at $600 \mathrm{xg}$ for $5 \mathrm{mins}$ at $4^{\circ} \mathrm{C}$. Cell pellets were resuspended in $50 \mathrm{uL}$ of cold lysis buffer (10mM Tris-HCl pH 7.4, $10 \mathrm{mM} \mathrm{NaCl}, 3 \mathrm{mM} \mathrm{MgCl}, 0.1 \%$ IGEPAL CA-630) and spin down immediately at $500 \mathrm{xg}$ for $10 \mathrm{mins}$ at $4^{\circ} \mathrm{C}$. Supernatant was discarded and nuclei were resuspended in 50uL transposition reaction mix (25uL 1x TD buffer fom Illuminia, $2.5 \mathrm{uL}$ Tn5 transposase, $22.5 \mathrm{uL} \mathrm{H}_{2} \mathrm{O}$ ), incubated at $37^{\circ} \mathrm{C}$ for $30 \mathrm{mins}$, and DNA was purified with a Qiagen MinElute kit (Qiagen). Library was amplified with KAPA HiFi HotStart Real-time PCR Master Mix (Roche) using indexed primers and sequenced on an Illumina Hiseq 2500 (paired-end 50 bp reads).

RNA-sequencing with Smart-seq. Smart-seq was performed as described previously ${ }^{34,57}$. Briefly, total RNA was isolated from naïve and activated B cells with Trizol (ThermoFisher) and the integrity of the RNA was accessed with TapeStation RNA Analysis ScreenTape or Bioanalyzer RNA pico kit (Agilent). 10ng of RNA was reverse transcribed using oligo-dT $\mathrm{dT}_{30} \mathrm{VN}$ primer in the presence of Template Switching Oligo (TSO) with SuperScript II reverse transcriptase. cDNA was pre-amplified with IS PCR primers and PCR products were cleaned up with Ampure XP beads. One ng of PCR product was used to generate library using NexteraXT library prep kit (Illumina) and tagmentated DNA was amplified for a 12 cycles PCR and purified with AmpureXP beads. Libraries were sequenced on an Illumina Hiseq 2500 with single-end 50 bp reads.

Statistical analyses. Statistical analyses and bar plots were performed and plotted with Prism 7 or R (v3.3.3). Bar graph and dot plots shown indicate mean and standard error. 


\section{Materials and Methods --- Bioinformatics analyses}

The reference genome used was mm10. Heatmaps and profile plots were generated using DeepTools ${ }^{58}$.

\section{CMSIP analysis}

Paired-end reads (50bp) were mapped to the mouse genome mm10 GRCm38 (Dec. 2011) from UCSC, using BSMAP (V.2.74) (-v 4 -R -n 1 -w 2 -r 0 -q $20-R-p ~ 8)^{59}$. Reads that mapped to the spike-in control (Lambda and Puro) were filtered out from the Sam file using awk. Tag directories were created with the remaining reads using makeTagDirectory from HOMER ${ }^{60}$ (-genome mm10 -tbp 1 -checkGC). Peaks were called with findPeaks from HOMER (-style histone -o auto -i). Peaks from all samples were merged with mergePeaks from HOMER into a master table. Quantile normalization was applied to all raw counts files and differentially enriched $5 \mathrm{hmC}$ regions were identified with edge ${ }^{61}$; a $p$ adjusted value of $\leq 0.05$ was used as a cutoff.

\section{H3K27Ac ChIP analysis}

Single end reads (50bp) were mapped to the mouse genome mm10 GRCm38 (Dec. 2011) from UCSC with Bowtie (V.1.1.2). Reads were sorted and PCR duplicates were removed using SortSam and MarkDuplicates, respectively from Picard Tools (V.2.7.1). Tag directories were created with makeTagDirectory (-genome mm10 -checkGC) from HOMER, and peaks were called with findPeaks (-region). Peaks from all samples were merged with mergePeaks from HOMER into a master table. Quantile normalization was applied to all raw counts files and differentially enriched $5 \mathrm{hmC}$ regions were identified with edge ${ }^{61}$; a $p$ adjusted value of $\leq 0.05$ was used as a cutoff.

\section{BATF ChIP analysis}

Single end reads (50bp) were mapped to the mouse genome mm10 GRCm38 (Dec. 2011) from UCSC with Bowtie (v1.1.2). Reads were sorted and PCR duplicates were removed using SortSam and MarkDuplicates, respectively from Picard Tools (V.2.7.1). Tag directories were created with makeTagDirectory (-genome mm10 -checkGC) from $\mathrm{HOMER}^{60}$, and peaks were called with findPeaks (-style factor -o auto).

\section{Definition of preferentially active enhancers}

Preferentially active enhancers (Supplementary Fig. 1h) were defined as distal H3K27Ac regions (> 1kb from TSS) that had ATAC-seq and H3K27Ac peaks overlapping in at least $50 \%$ of either peak/region; overlapping was calculated with intersectBed -f 0.5 -f 0.5 -e (Bedtools v2.26.0). The differential enrichment in an enhancer was called if it contains a differentially enriched H3K27Ac region as well as at least one differentially access region.

\section{ATAC-seq analysis}

Paired-end reads (100 bp) were mapped to the mouse genome mm10 GRCm38 (Dec. 2011) from UCSC using Bowtie 1.0.0 ("-p 8 -m 1 --best --strata -X 2000 -S --fr --chunkmbs 1024."). Reads that failed this alignment step were filtered for Illumina adapters and low quality using "Trim Galore!" ("--paired --nextera --length 37 --stringency 3 --three_prime_clip_R1 1 --three_prime_clip_R2 1") and re-mapped using the same parameters. Both mapping results were merged and processed together to remove duplicates using picard-tools-1.94 MarkDuplicates. Mitochondrial and Chromosome $Y$ reads were excluded.

Subnucleosomal fragments were obtained with SAMtools and awk to identify DNA fragments that were less than or equal to $100 \mathrm{nt}$ in length. These fragments were used to call peaks using HOMER (v4.9.1) findPeaks function for each replicate ("-size 500 -region -center -P .1 -LP .1 -poisson .1 -style dnase") and all the peak sets were merged to generate a global set. Peaks overlapping with ENCODE blacklisted regions ${ }^{62}$ were removed. From each sample, Tn5 insertion sites were obtained by isolation of the initial $9 \mathrm{bp}$ of mapped reads ${ }^{63}$ which were used to compute the number of transposase insertions per peak using MEDIPS ${ }^{64}$. Raw reads from all samples were quantile-normalized prior to differential coverage analysis using edgeR without TMM (Trimmed mean of M-values) normalization. Only regions with more than 32 normalized reads across the samples per comparison. Differentially accessible regions were defined by an adjusted $p$ value (FDR) lower than 0.05 and a log 2 fold enrichment higher equal than 1 . 


\section{OxBS analysis}

OxBS-seq reads were mapped to both the mouse genome mm10 GRCm38 (Dec. 2011) from UCSC and the phage Lambda genome (GenBank: J02459.1) using bsmap-2.90 (" -v 15 -w 3 -p 4 -S 1921 -q 20 -A AGATCGGAAGAGC -r $0-R-V 2$ "). The mapping results were separated into reads belonging to the mm10 genome and each of the three loci from lambda used for oxidation and conversion efficiency calculation. Methylation calls from lambda- and mm10-derived reads were obtained using bsmap-2.90 function methratio.py (" - u -p -g -i "correct" -x CG,CHG,CHH "). Conversion efficiencies as well as posterior probabilities of methylation, hydroxymethylation and unmodified cytosine were calculated by luxGLM v.0.666 (prior probabilities used for for C, hmC and mC "998,1,1", "6,2,72" and $" 1,998,1$ " respectively $)^{65}$. Following genomic positions from lambda used for oxidation and BS treatment efficiencies: chrLambda:22893-23053 C; chrLambda:23765-23925 hmC; chrLambda:47335-47495 mC.

\section{WGBS analysis}

WGBS reads were mapped to both the mouse genome mm10 GRCm38 (Dec. 2011) from UCSC. Bisulfite conversion efficiency was estimated based on cytosine methylation in non-CpG context. For all the samples the bisulfite conversion efficiency was higher than 0.9996. Duplicated reads caused by PCR amplification were removed by BSeQC (v1.2.0) applying a $p$ value cutoff Poisson distribution test in removing duplicate reads $(1 \mathrm{e}-5)^{66}$. Consequently, a maximum of three stacked reads at the same genomic location were allowed and kept for further analysis. In addition, BSeQC was employed for removing DNA methylation artifacts introduced by end repair during adaptor ligation. Overlapping segments of two mates of a pair were reduced to only one copy to avoid considering the same region twice during the subsequent DNA methylation quantification. To estimate CpG DNA methylation at both DNA strands, methratio.py script was executed from BSMAP (v2.90) (-u -r -z -g -i "correct" -x CG). To identify differentially methylated cytosines and regions (DMCs and DMRs), a naïve B cells dataset and was compared to a activated B cell replicate using RADmeth methpipe-3.4.2 (adjust -bins 1:100:1; merge - $p 0.05)^{67}$.

\section{RNA-seq analysis}

RNA-seq samples at four different time-points collected from WT and DKO conditions were first mapped to the mouse genome mm10/GRCm38 using both Hisat2 ${ }^{68}$ ("--no-mixed --no-discordant --add-chrname -dta") and Tophat $^{69}$ ("--no-novel-juncs") alignment programs separately. Aligned bam files obtained from both the programs were further used to generate the Hisat2- and Tophat2-specific counts using HTseq-count program ${ }^{70}$ (default parameters). Hisat2- and Tophat2-specific count files at each time point for WT and DKO conditions were then used to identify the differentially expressed genes (FDR < 0.05) between matching time points using edgeR program $^{61}$. Potential batch effects were removed using svaseq program ${ }^{71}$. Finally, the common differentially expressed genes obtained from both Hisat2- and Tophat2-specific list were used to perform the downstream analysis.

\section{Genome-browser track generation for ChIP-seq}

ChIP-seq results from Tet2, Ig control, E2A, PU.1, p300, and GCN5 were processed as follow to generate the genome browser tracks. Fastq files were mapped to mm10 reference genome with Bowtie 2 (v2.1.0) with "-verysensitive". The mapped SAM files were converted to BAM using Samtools ( $\mathrm{v} 1.7)$ view $-\mathrm{h}-\mathrm{F} 4$, and duplicates were removed using Picard (v2.7.1). BigWig files were made by first generating a BedGraph files from the filtered Bam files using Bedtools (v2.26.0) genomecov followed by bedGraphToBigWig (v4) with read counts normalized to $10,000,000$ reads.

\section{Miscellaneous analyses of regions}

For distance between regions to the closest TSS was analyzed with HOMER software with "annotatePeaks.pl annStats". Overlap between regions was analyzed by "bedtools intersect" with no requirement for the degree of overlapping. The degree of significance for overlap between superenhancers and test regions was estimated by Fisher exact test using "bedtools fisher".

\section{Time-series analysis}


For a unified analysis of the RNA-seq time-course data (0hr to 96hr) from WT samples, TCseq package ${ }^{72}$ was used on the combined RNA-seq read counts, obtained after applying Tophat ${ }^{69}$ and Hisat ${ }^{68}$ alignment programs (Described in the previous RNA-seq analysis part). TCseq utilizes GLM method implemented in edgeR package ${ }^{61}$ to detect the differential events in gene expression. Differential analysis was performed between "Ohr" to the rest of the time points, and the significant differential events were extracted whenever a log2FC $>2$ or $<-2$ and FDR $<0.05$ criteria was satisfied. To detect the temporal pattern of the differential gene expressions (RPKM values), a soft clustering algorithm implemented in TCseq program was applied ("algo $=$ ' $\mathrm{cm}$ ', $\mathrm{k}=6$, standardize $=$ TRUE"). Finally, the differential genes were assigned to a cluster (C1- C6) representing a specific temporal pattern of expression, if the membership probability of the genes to a cluster is 0.5 or more.

\section{Published datasets}

Naïve H3K4me1 (Oh): SRR1535686, SRR1535685. Activated H3K4me1 (48h): SRR1014530. SRR1087900. Naive WGBS (0h): SRR1003257. Activated WGBS (48h): SRR1020523. Naive PU1 (0h): SRR2976278. Activated PU1 (48h): SRR1014532. Naïve DSG control (0h): SRR3158132. Activated E2A DSG anti-CD40/IL-4 (48h): SRR3158146. Naïve Brg1 (Oh): SRR3619348. Naïve Chd4 (Oh): SRR3619349. Naïve Gcn5 (Oh): SRR3619350. Naïve p300 (0h): SRR3619356. Activated Brg1 (24h): SRR3619334. Activated Chd4 (24h): SRR3619335. Activated Gcn5 (24h): SRR3619336. Activated p300 (24h): SRR3619342. 
a

b

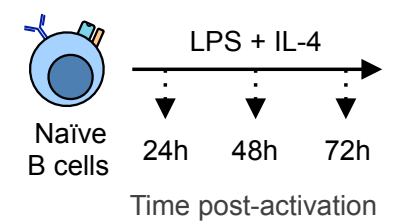

$5 \mathrm{hmC}$ regions at

$72 \mathrm{~h}$ vs naïve

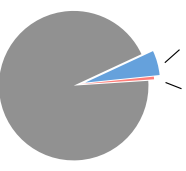

$n=159,305$
$8,454(5.3 \%)$

$928(0.6 \%)$

Common

Increase

Decrease

d
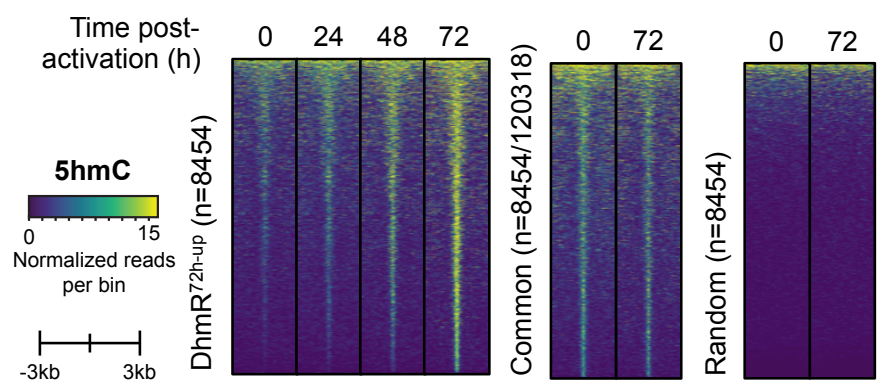

f
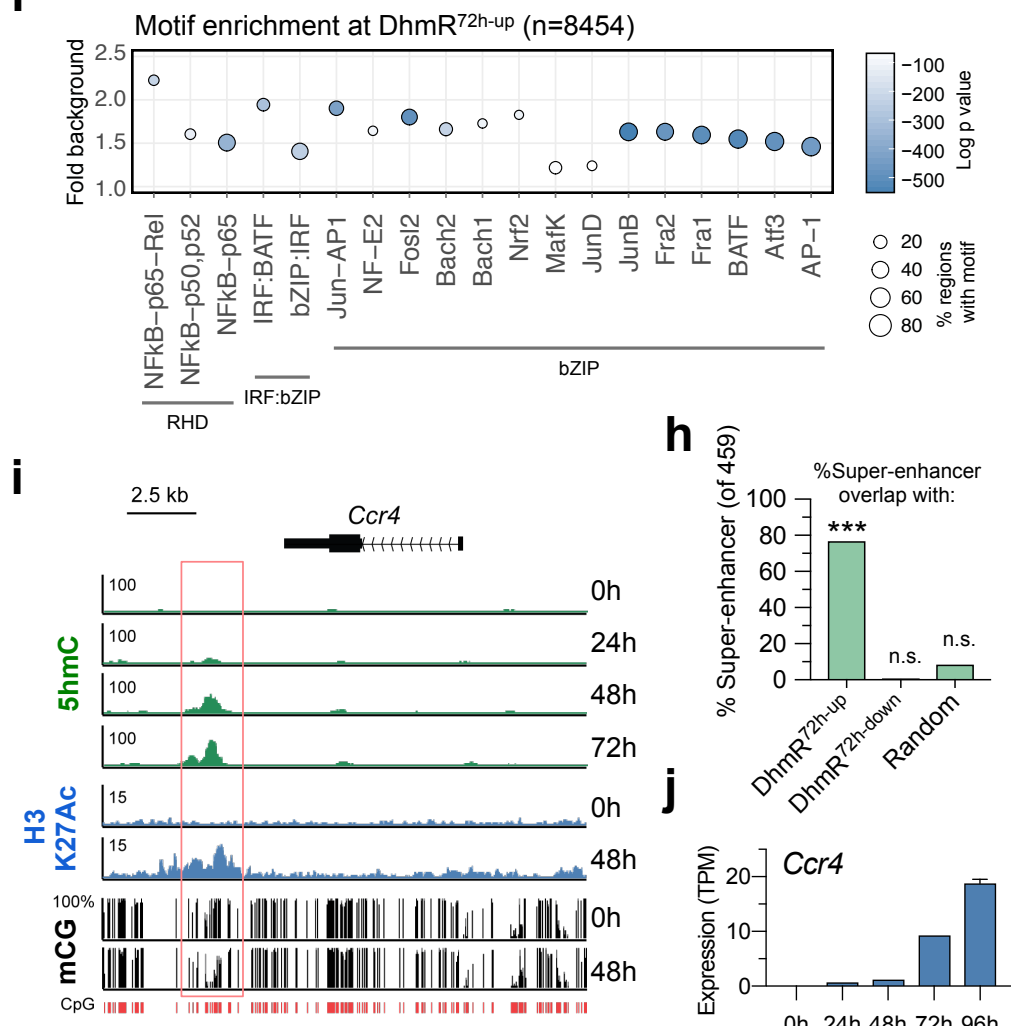

Oh $24 \mathrm{~h} 48 \mathrm{~h} 72 \mathrm{~h} 96 \mathrm{~h}$

C Differential $5 \mathrm{hmC}$ region vs naïve (DhmR)

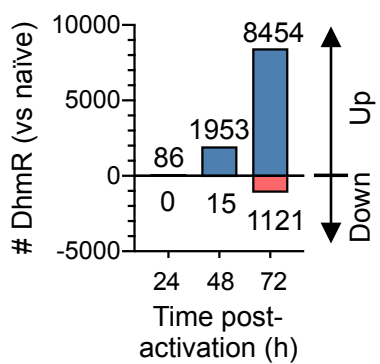

e

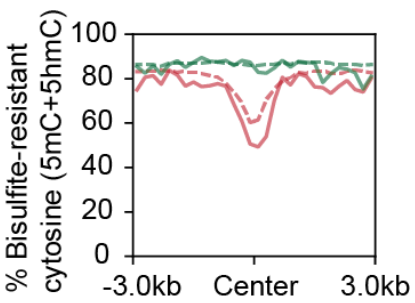

$\operatorname{DhmR}^{24 h-u p}(\mathrm{n}=85)$

- \%meth in WT-naïve

— \%meth in WT-48h

DhmR ${ }^{48 h-u p}$ ( $\left.n=1953\right)$

.... \%meth in WT-naïve

.... \%meth in WT-48h

g
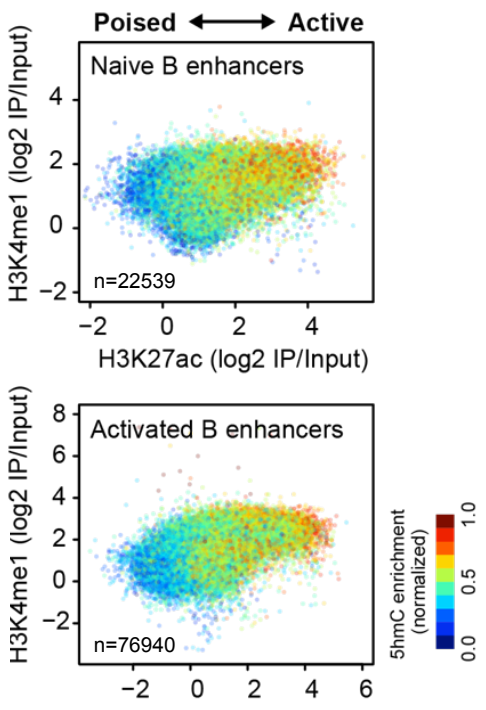

H3K27ac (log2 IP/Input)

Figure 1. 
a

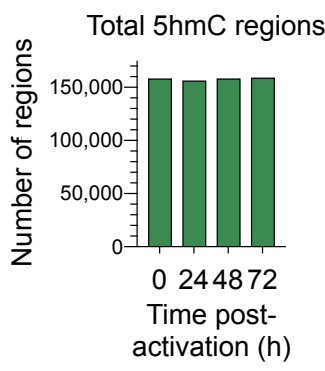

d

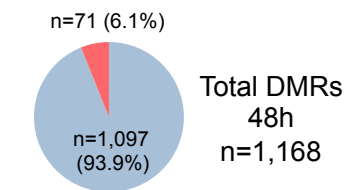

Decreased mCG

Increased mCG

f

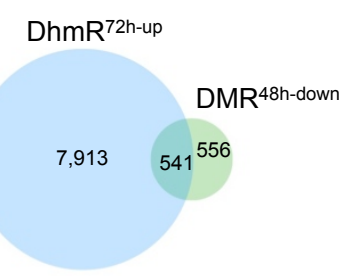

b
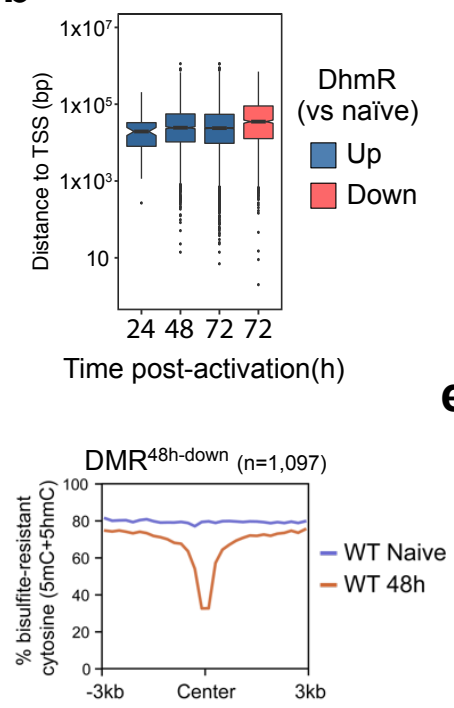

g

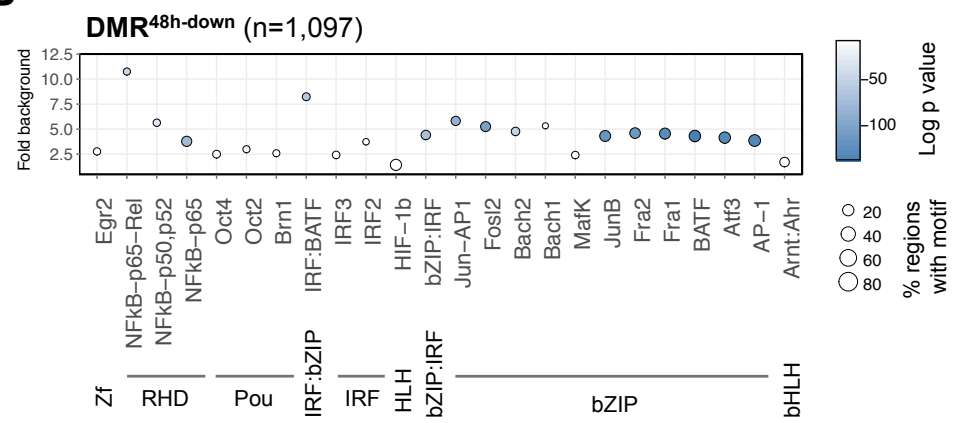

C

Time post-activation (h)

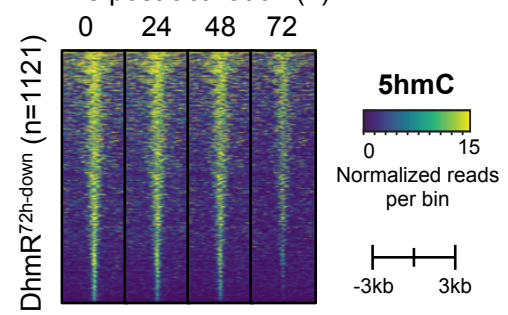

e Time post-activation (h)

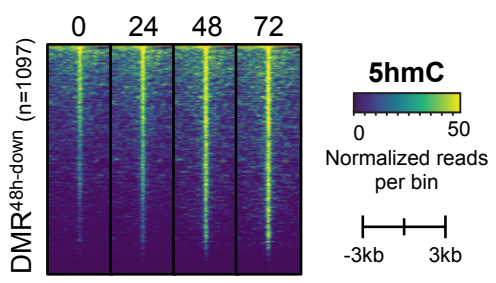

h
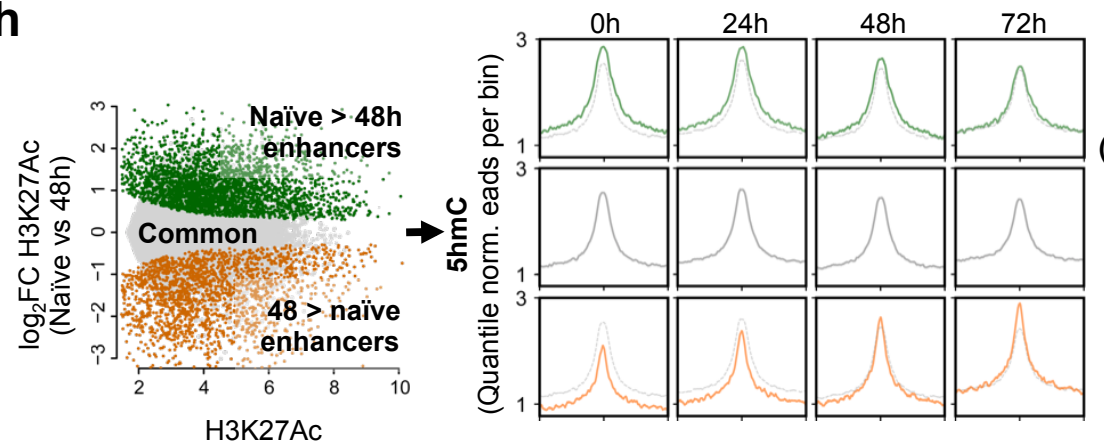

Active enhancers (H3K27Achi accessibility ${ }^{\text {hi) }}$

(mean quantile norm. reads)

Fig. S1. 
a

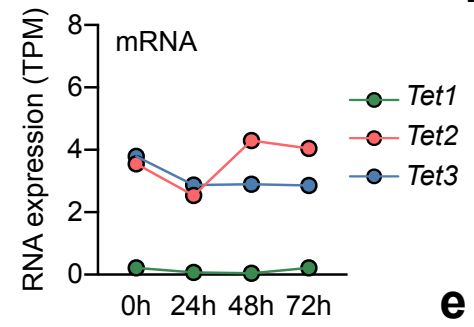

C

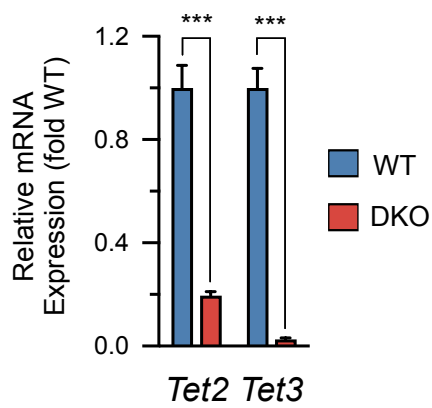

d

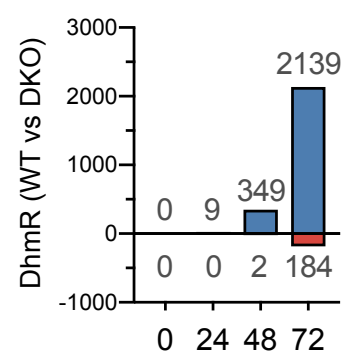

Time post-activation $(\mathrm{h})$

DhmR WT>DKO

DhmR DKO>WT

f b

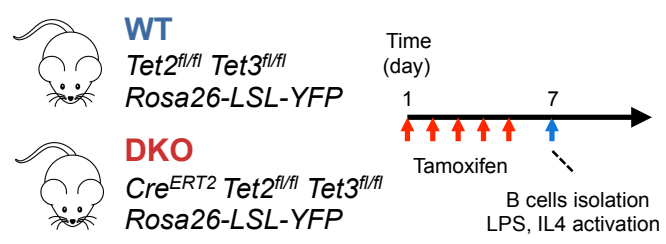

Differential hydroxymethylated regions (DhmRs) at $72 \mathrm{~h}$
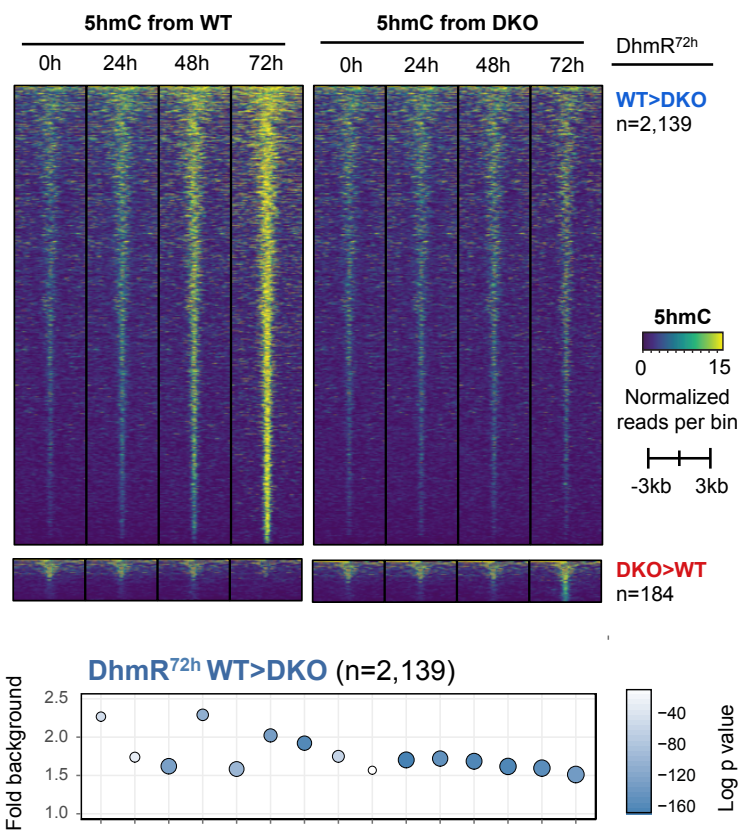

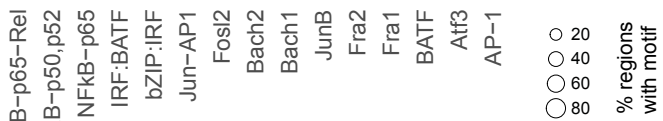

竞

咅崖苜F

bZIP

RHD

Figure 2. 
a

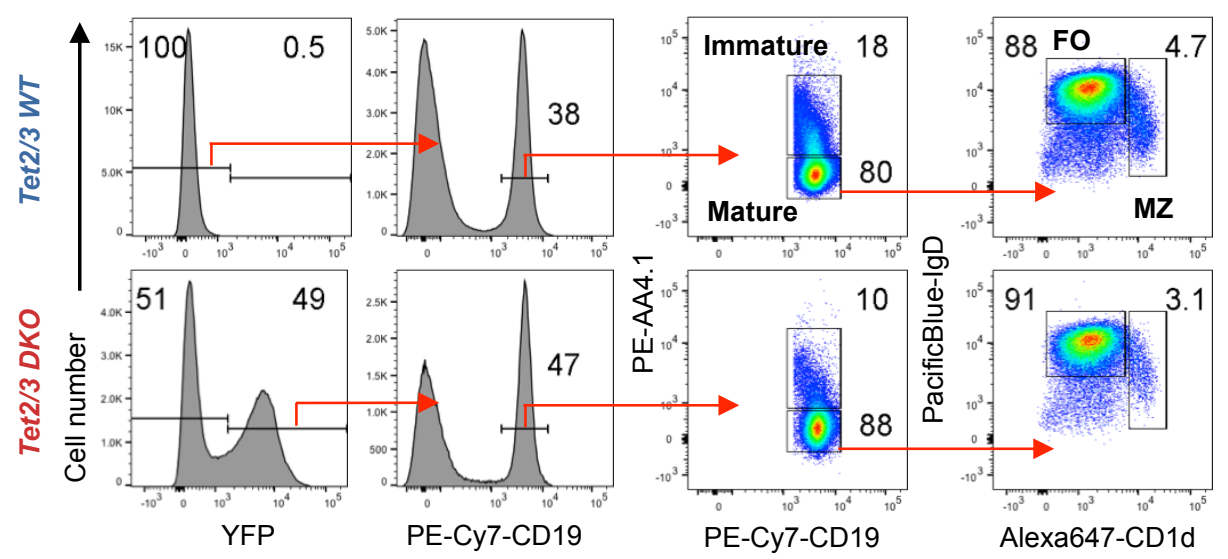

b

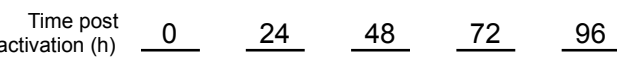

d

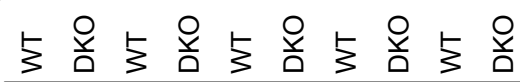

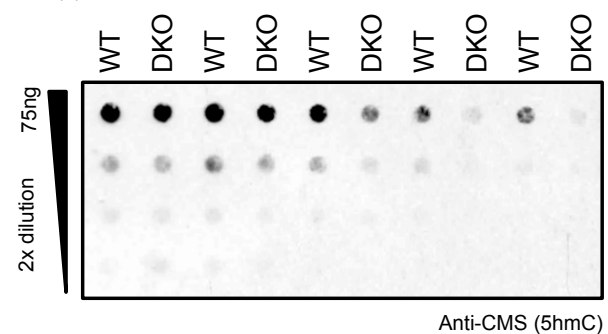

DNA methylation from

C

DhmR ${ }^{72 h}$ WT $>$ DKO DhmR ${ }^{72 h}$ DKO $>$ WT $(n=2139) \quad(n=184)$

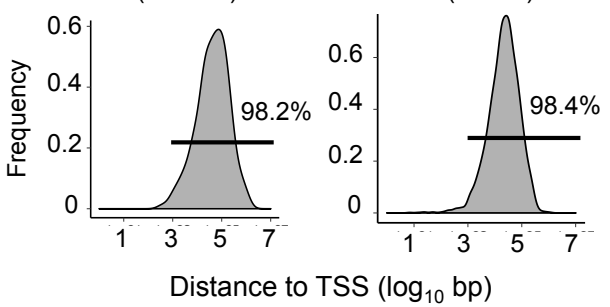

DKO>WT
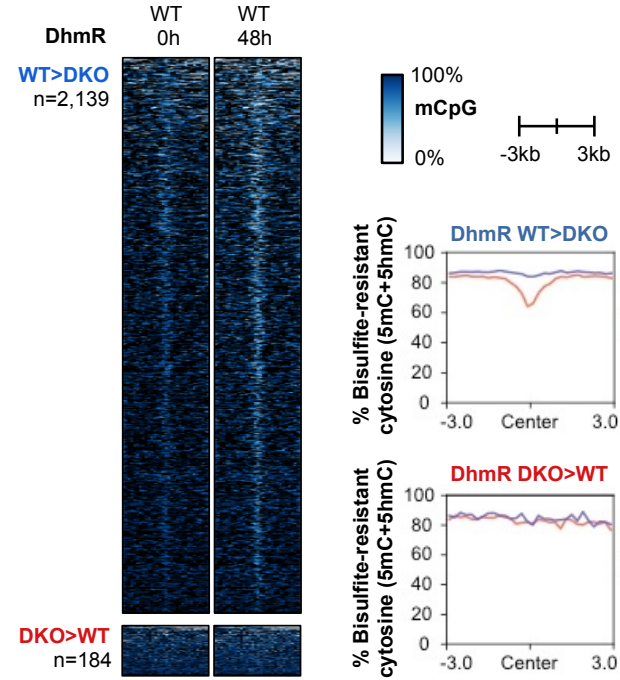

$0 \% \quad-3 \mathrm{~kb} \quad 3 \mathrm{~kb}$
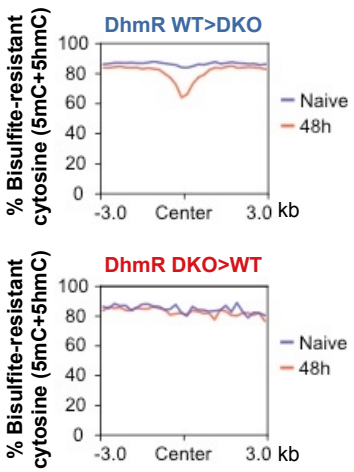

Fig. S2. 
a

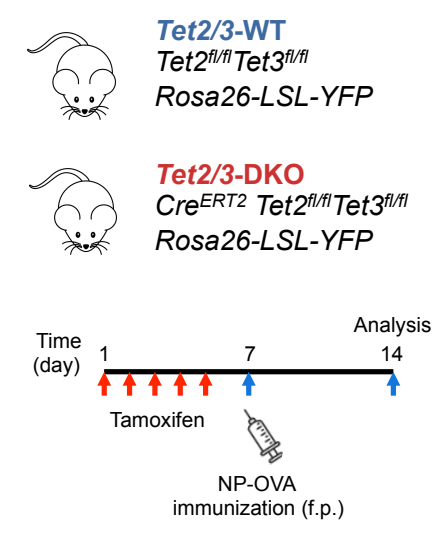

g

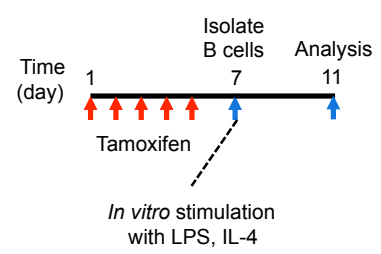

k

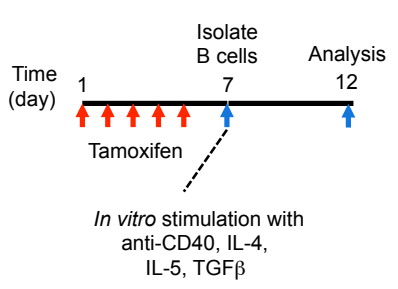

b
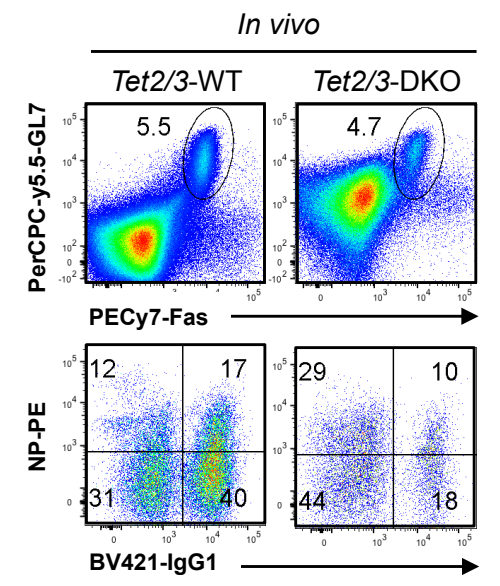

h

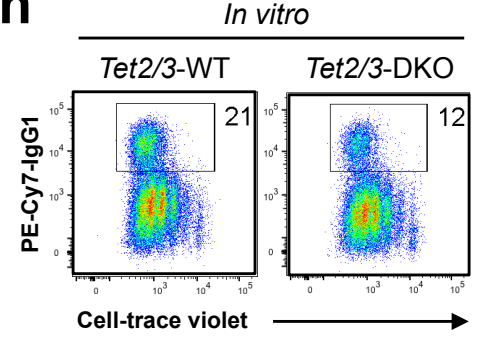

I

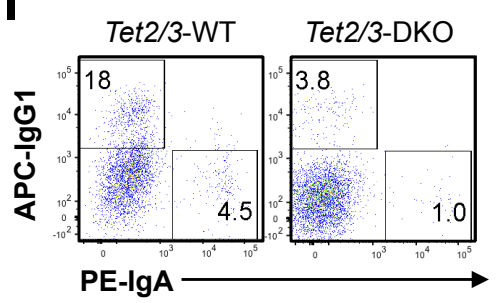

c d

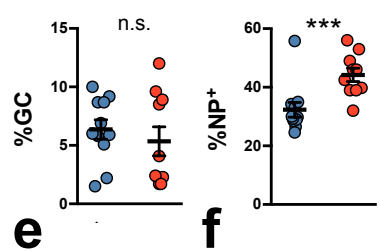

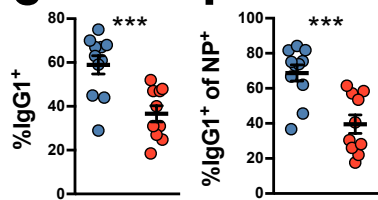

o Tet2/3-WT o Tet2/3-DKO

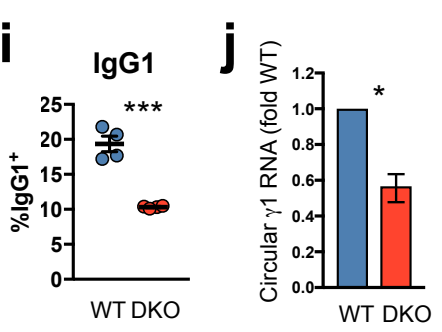

m

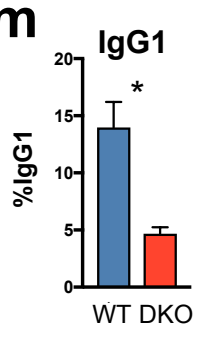

n

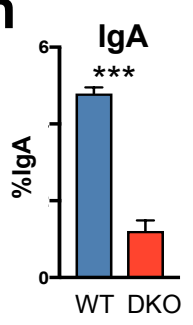

Figure 3. 
a

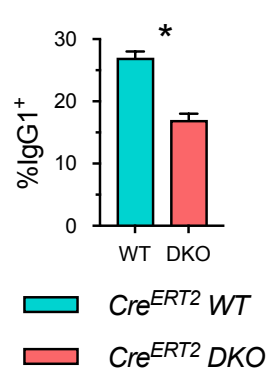

d

g

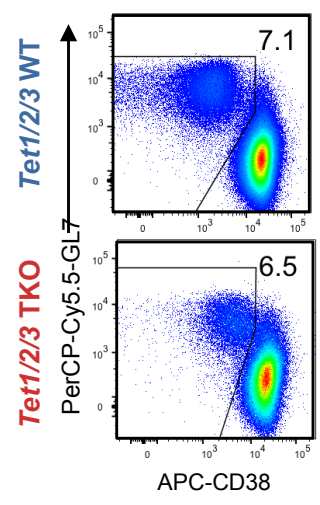

b

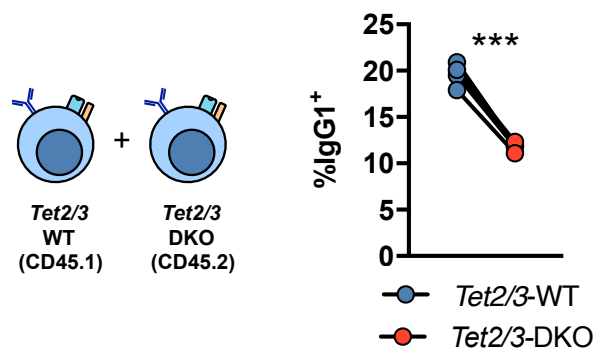

C

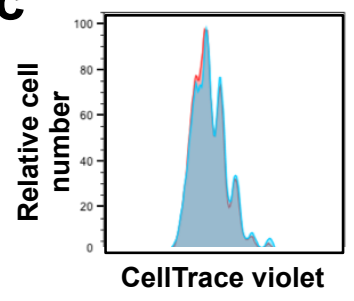

TET2/3-WT

TET2/3-DKO e

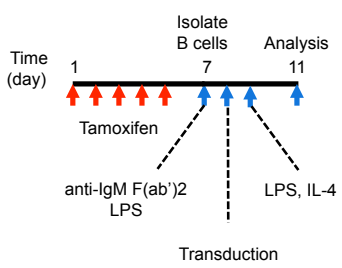

DKO

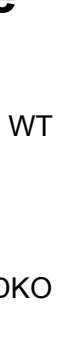

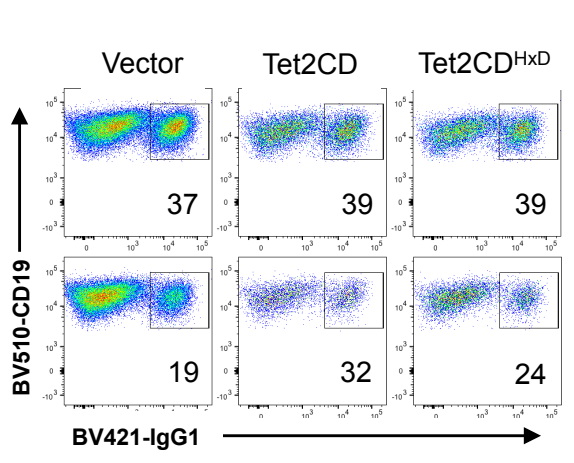

f

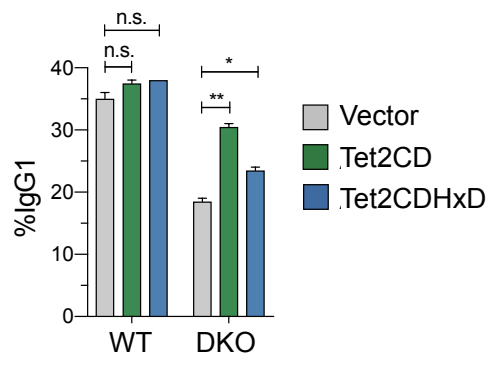

h

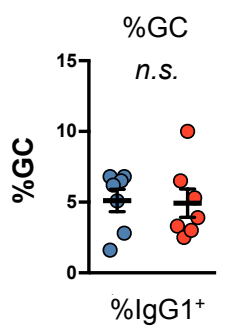

$\%$ NP-specific

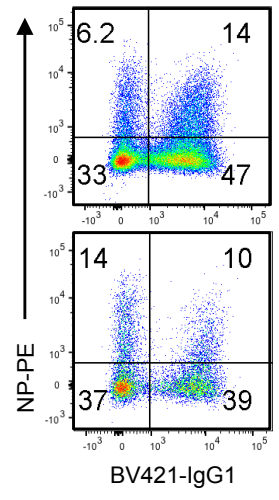

O Tet1/2/3-WT

O Tet1/2/3-TKO

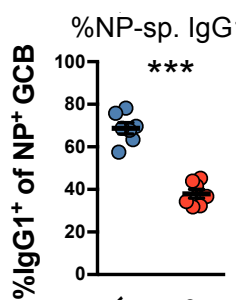

Fig. S3.
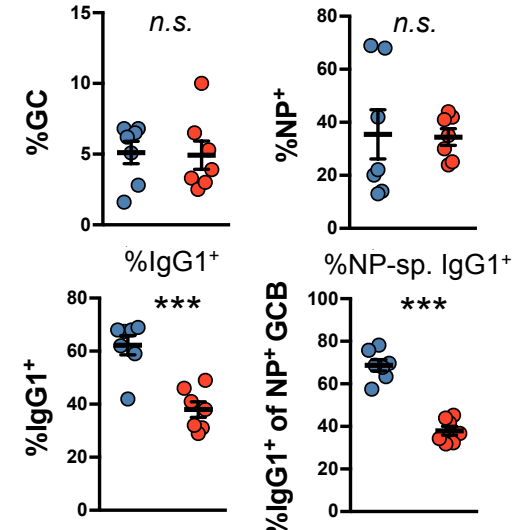

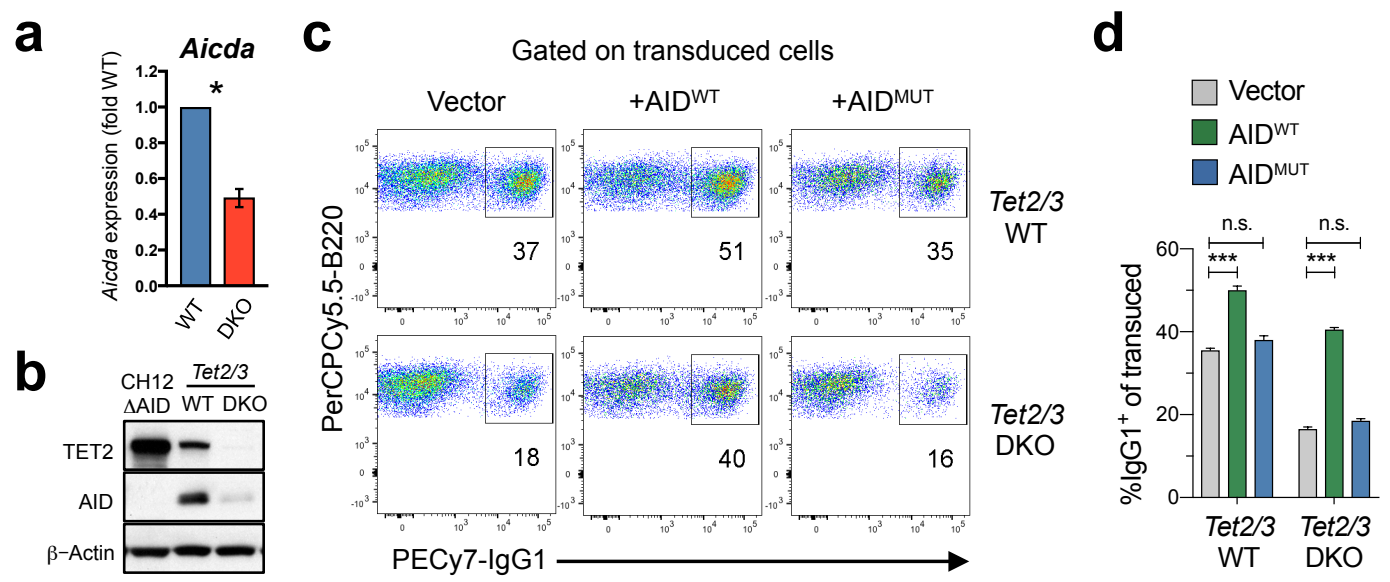

\section{Figure 4.}


a

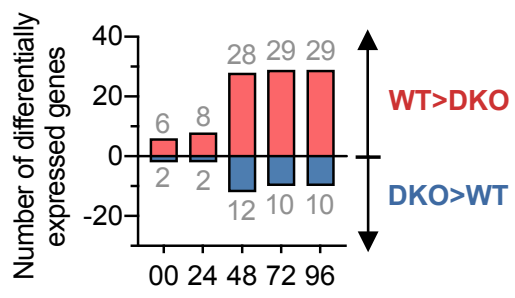

Time post-activation (h)

C

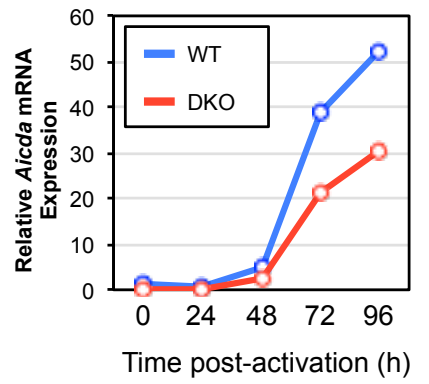

j
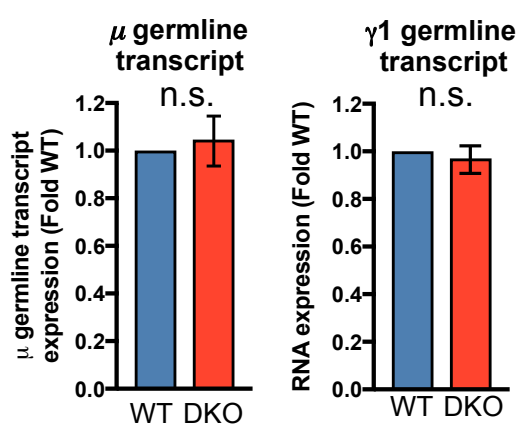

d
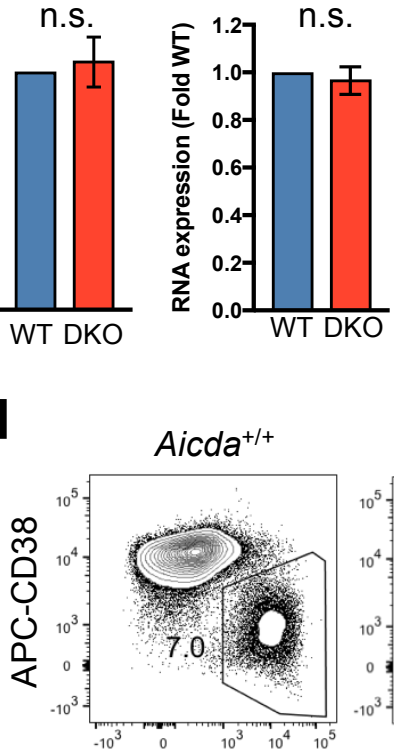

b
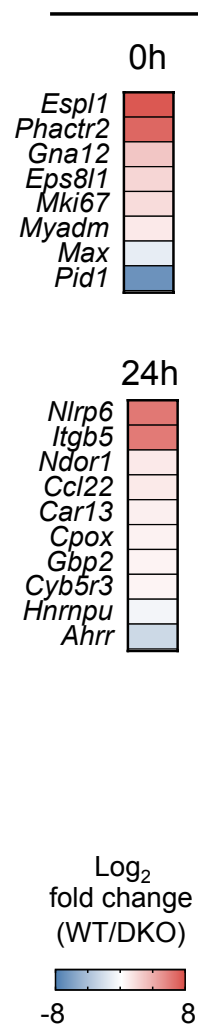

Aicda $^{+/ C r e}$

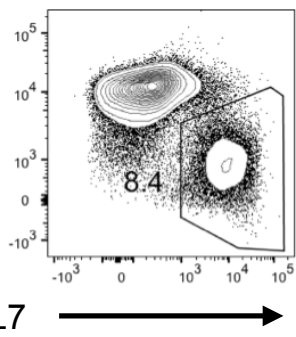

f

PerCP-Cy5.5-GL7

f
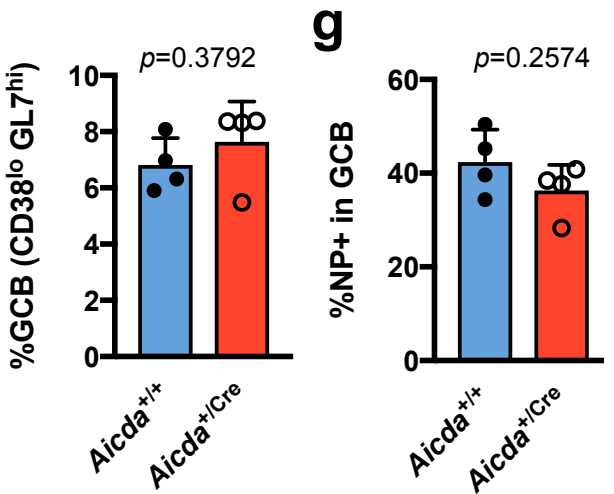

Differentially expressed genes (WT/DKO)

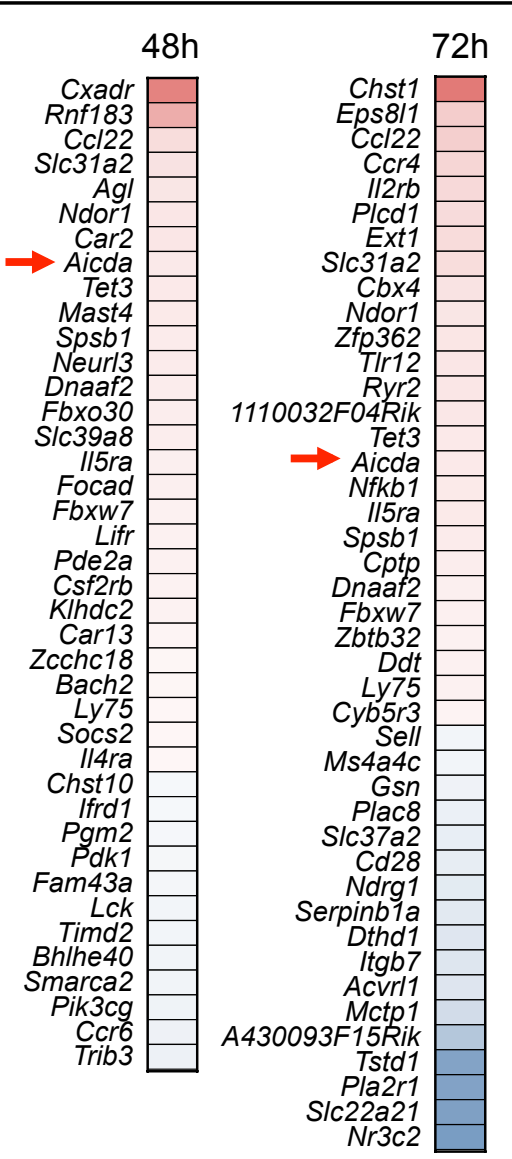

$96 \mathrm{~h}$

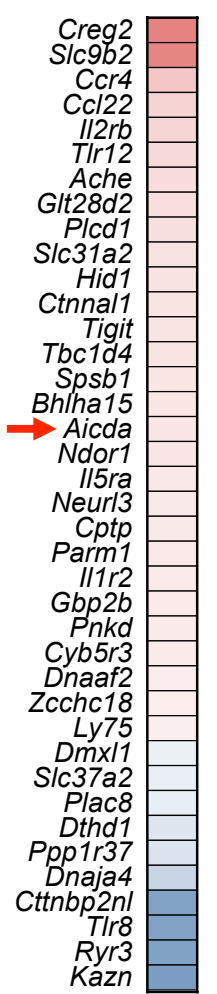

Fig. S4.

e

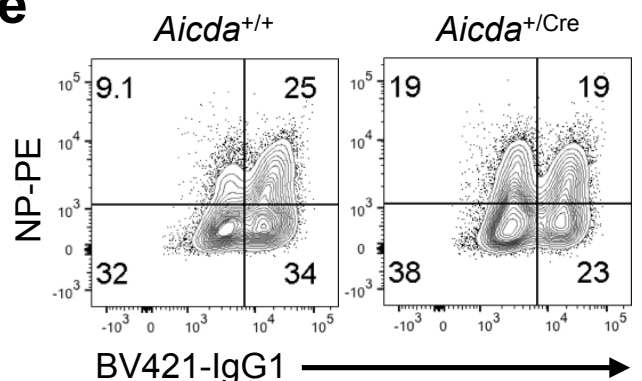

$\mathrm{h}$
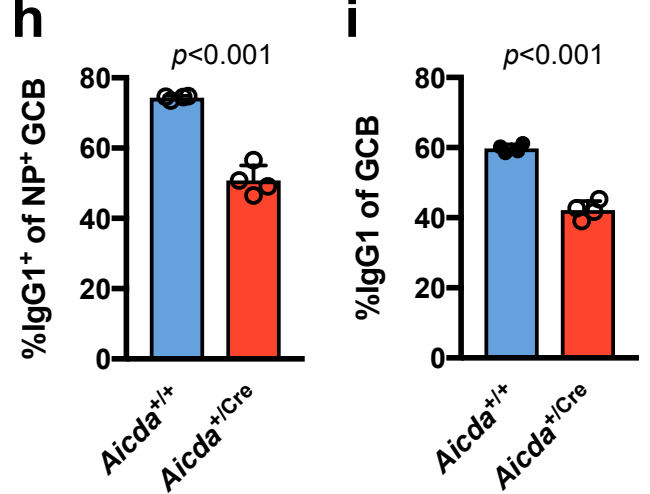
a

$-26 \mathrm{~kb}-21 \mathrm{~kb}$

$-8 \mathrm{~kb}$

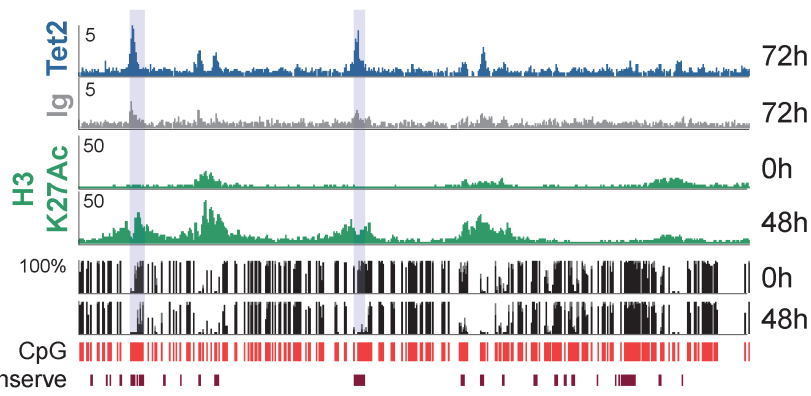

"super-enhancer"

b

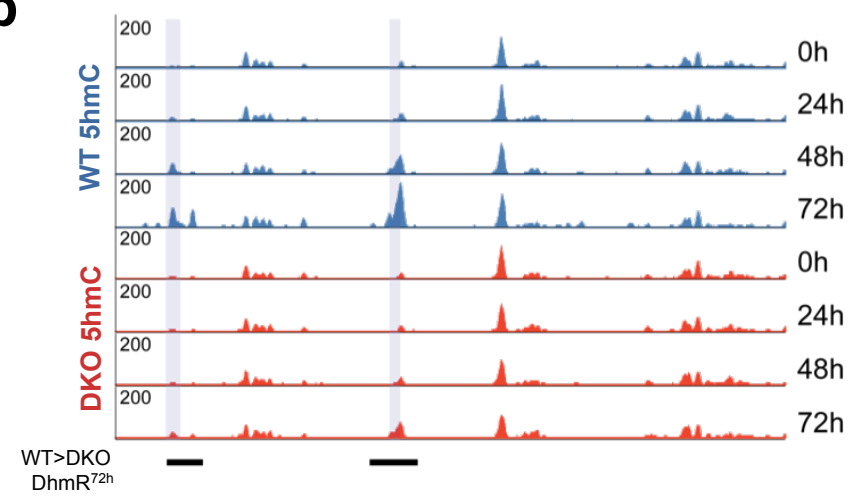

C
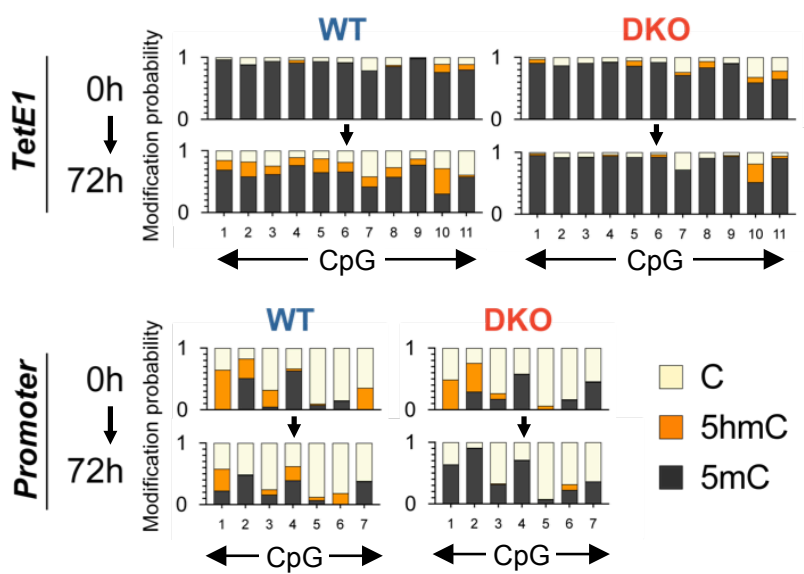

$\square$ C

$5 \mathrm{hmC}$

$5 \mathrm{mC}$

Figure 5. 
a

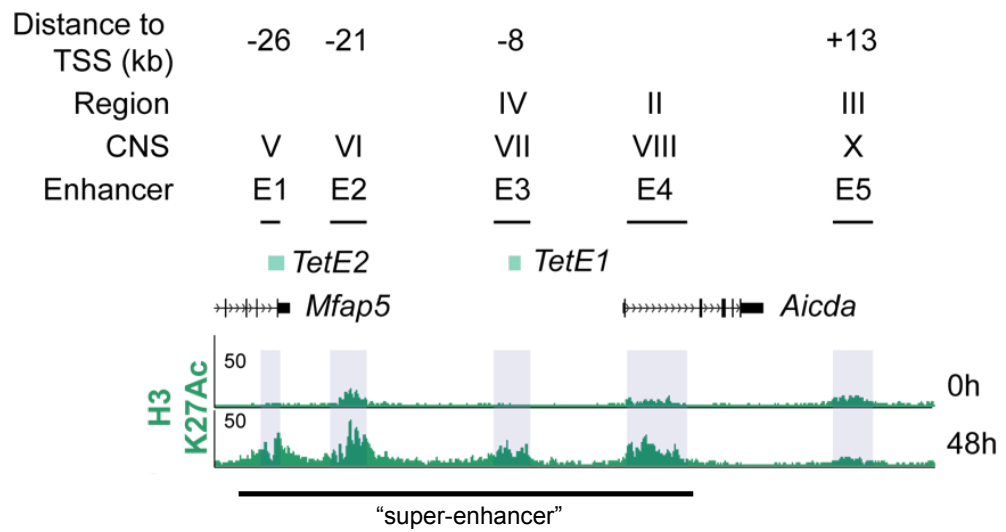

b

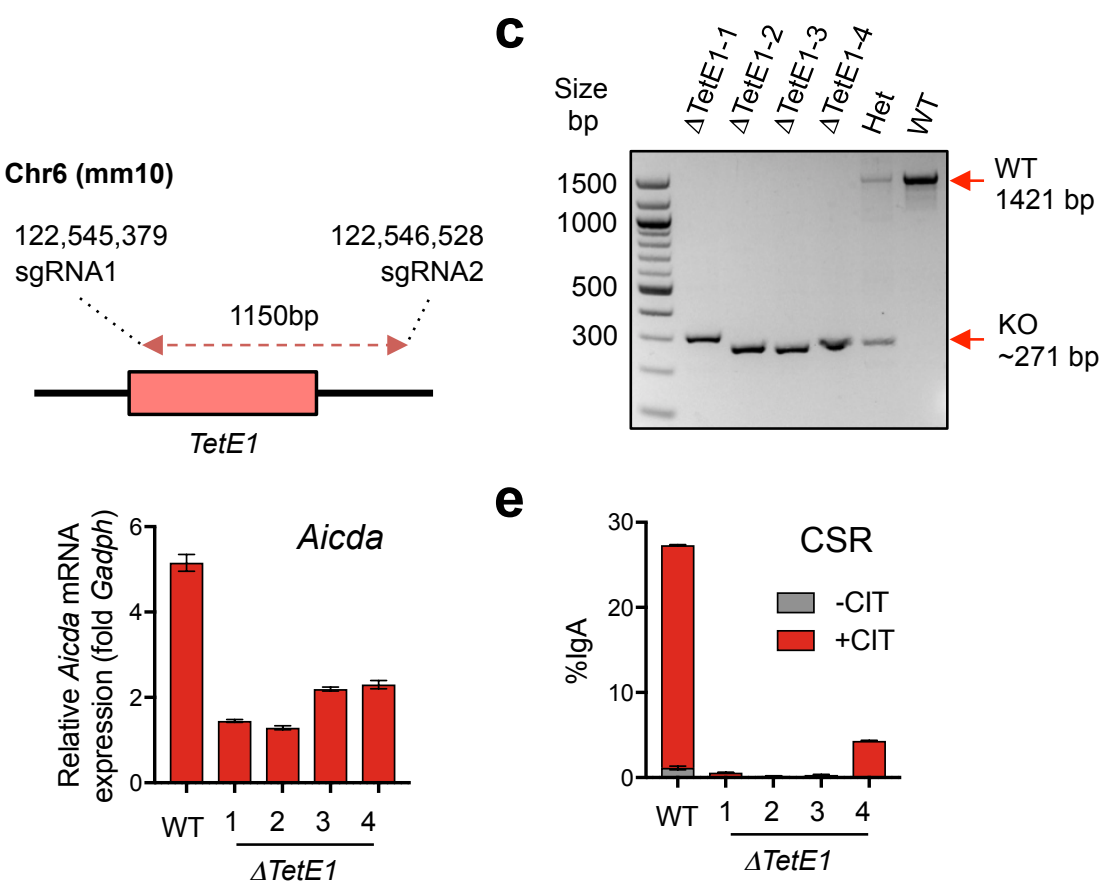

Fig. S5. 
a
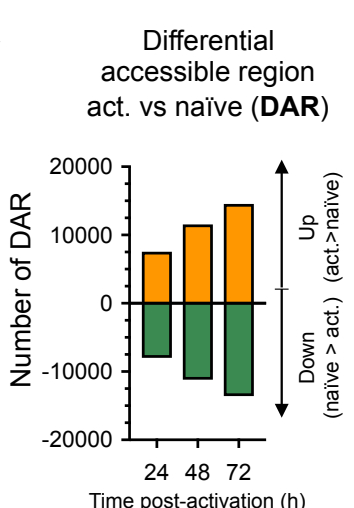

d

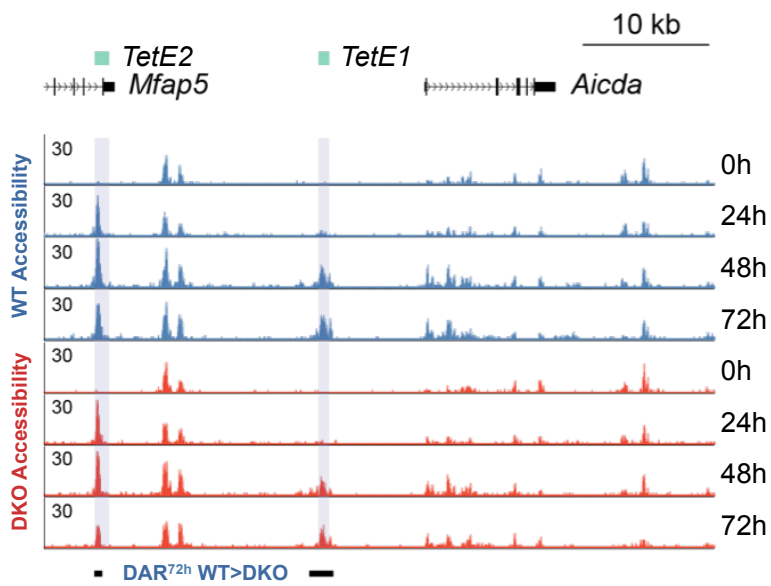

b

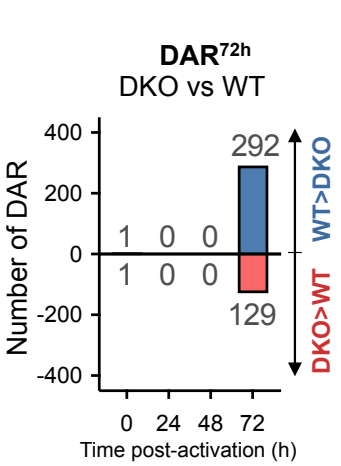

C

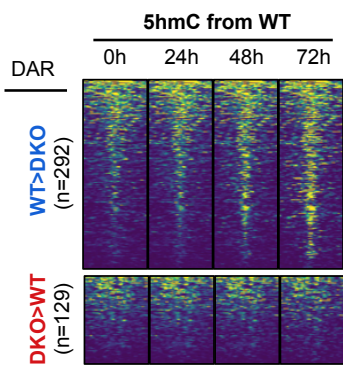

\begin{tabular}{cccc}
\multicolumn{4}{c}{ 5hmC from DKO } \\
\hline Oh & $24 \mathrm{~h}$ & $48 \mathrm{~h}$ & $72 \mathrm{~h}$
\end{tabular}
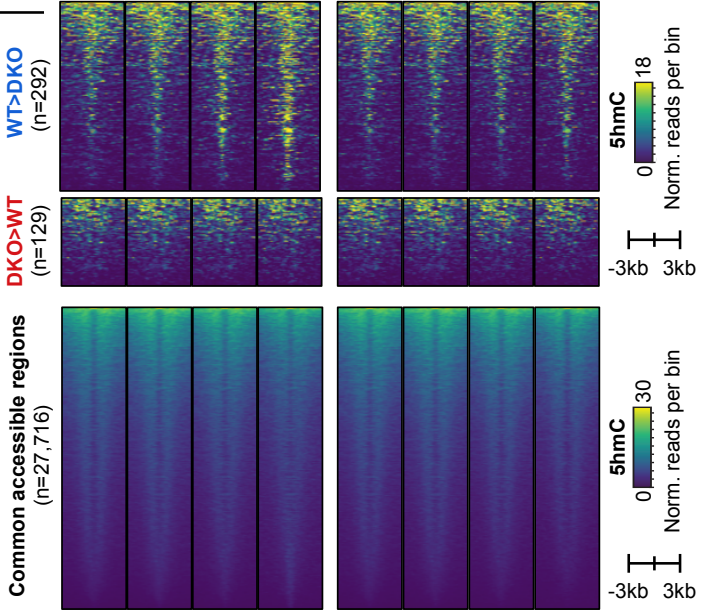

e

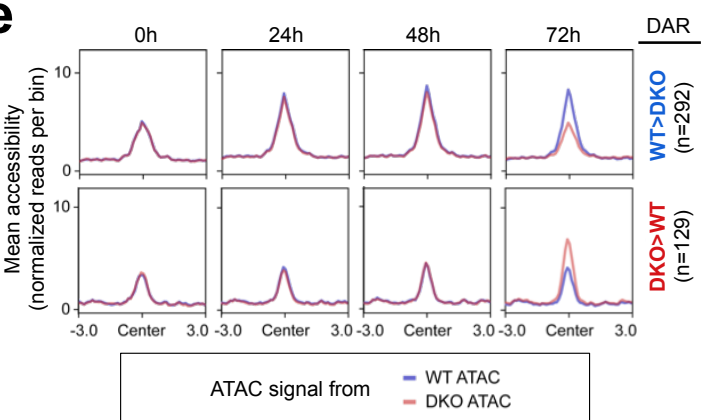

Figure 6. 
a

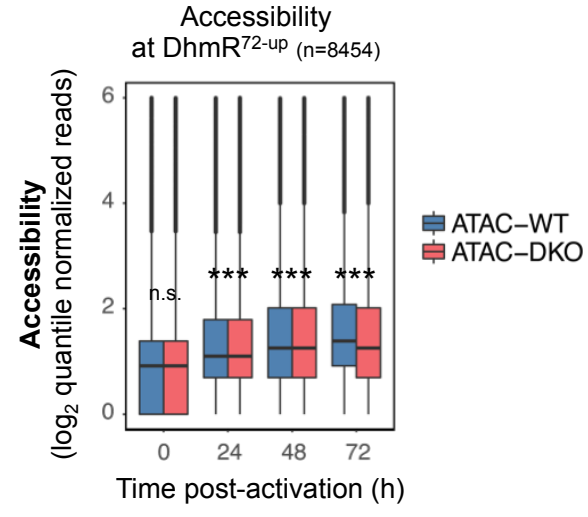

b
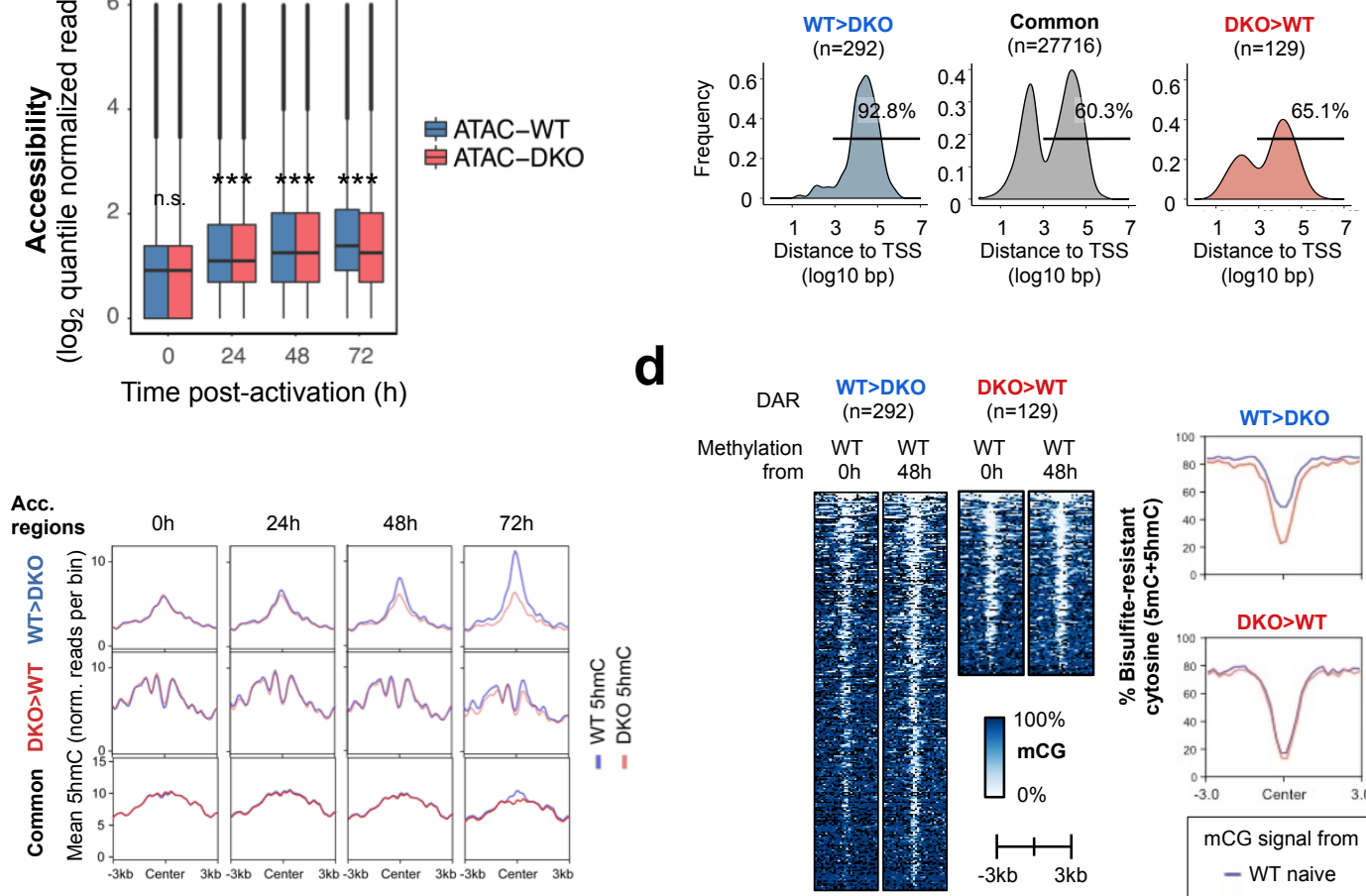

$\begin{gathered}\text { DKO }>\text { WT } \\ (n=129)\end{gathered}$
WT $\quad$ WT
Oh $\quad 48 h$
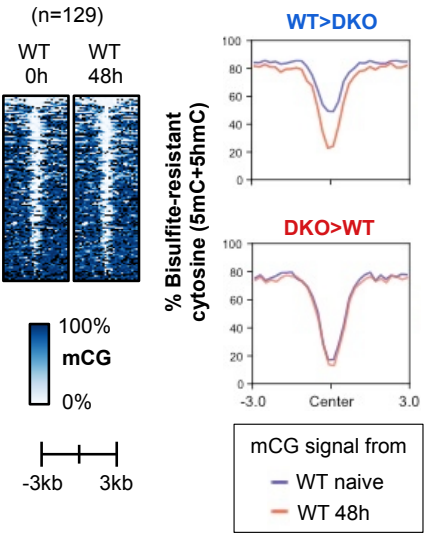

e

f

DAR $^{72 h}$ WT $>$ DKO $(n=292)$

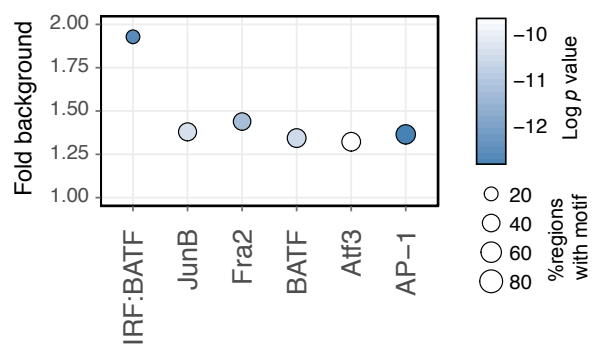

$D^{72 h}$ DKO $>W T(n=129)$

No significant enrichment

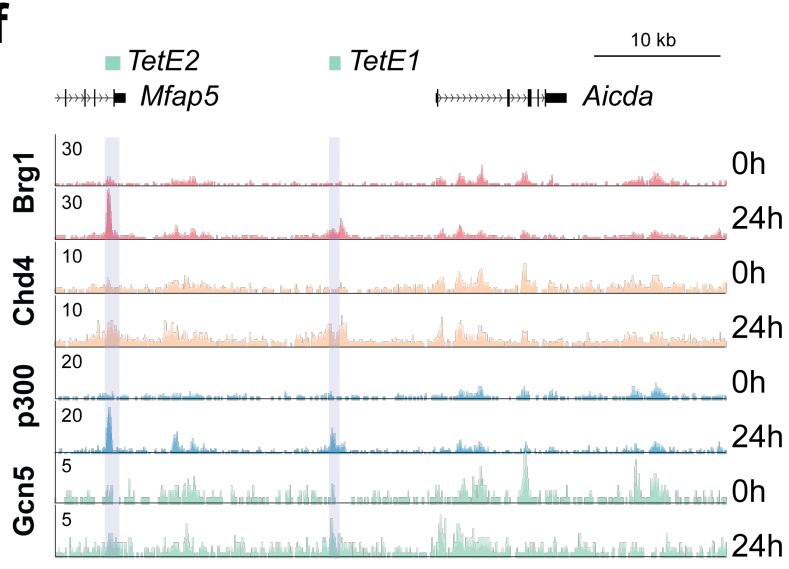

Fig. S6. 


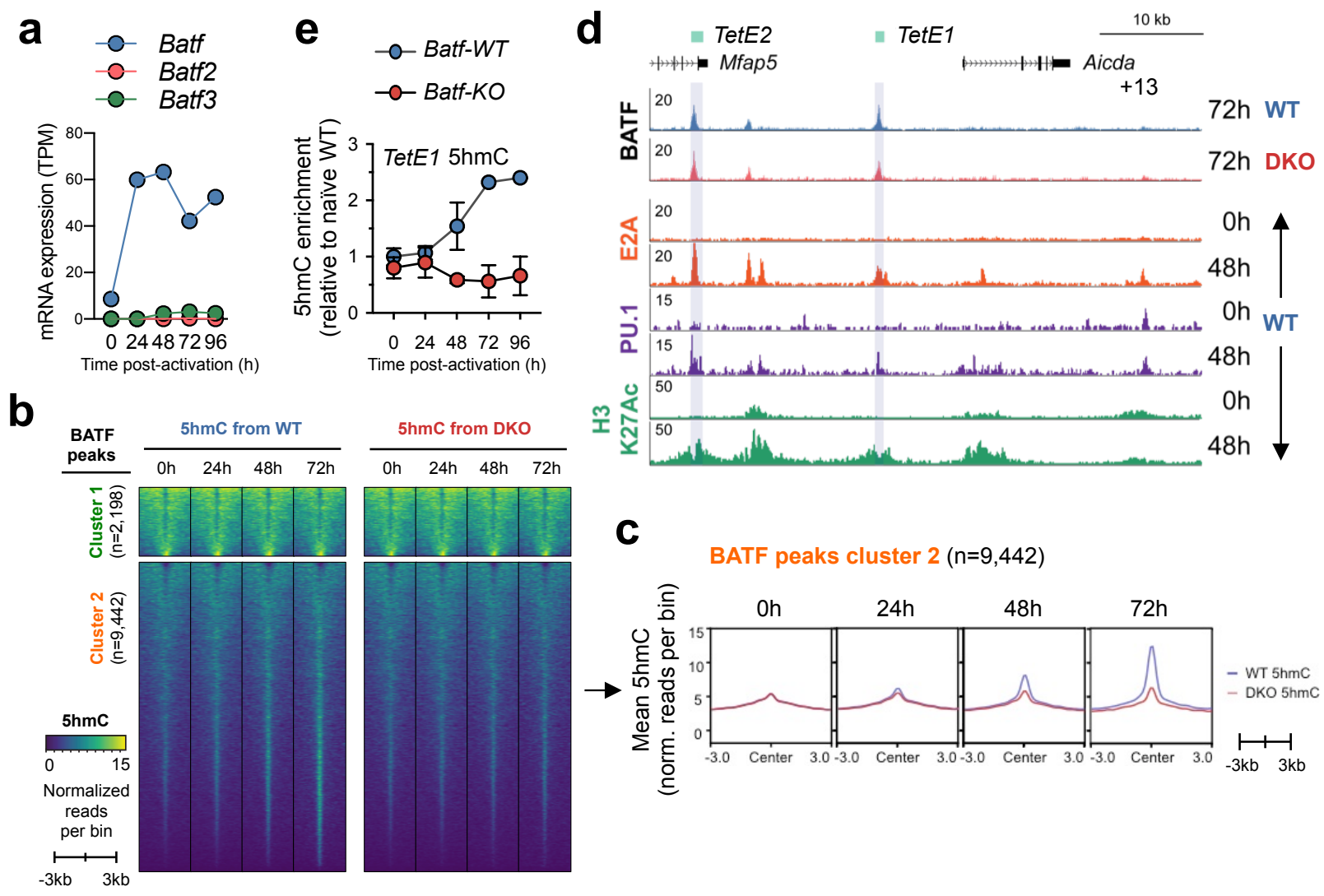

Figure 7. 
a

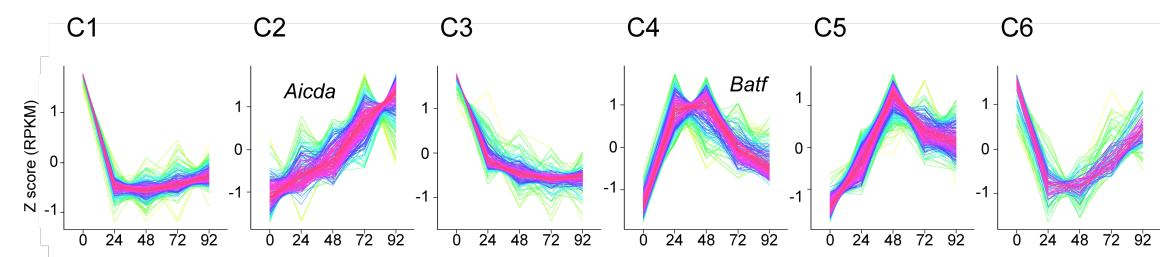

Time post-activation (h)

b

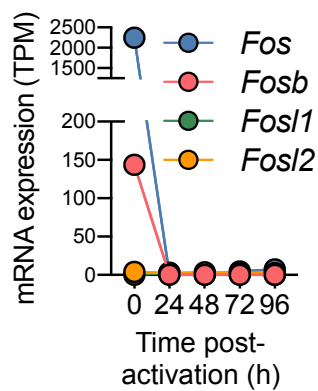

C

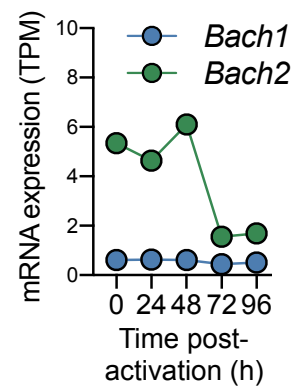

d

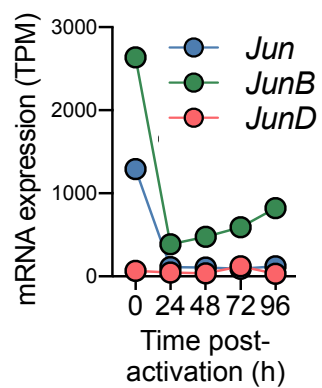

e
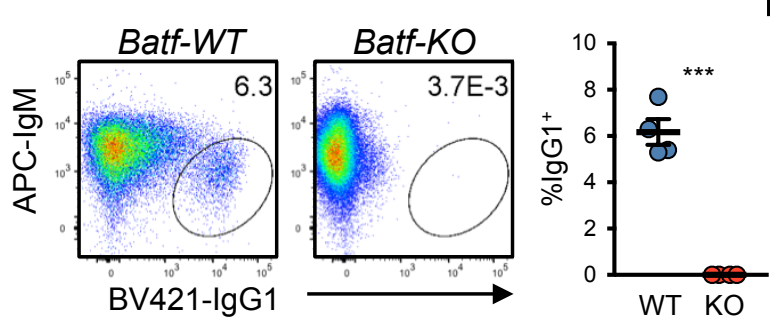

f

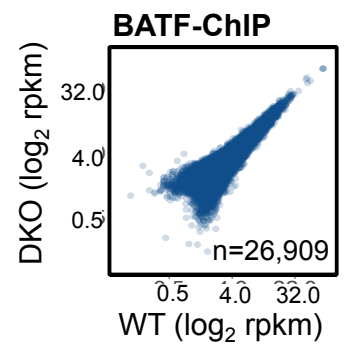

g

h
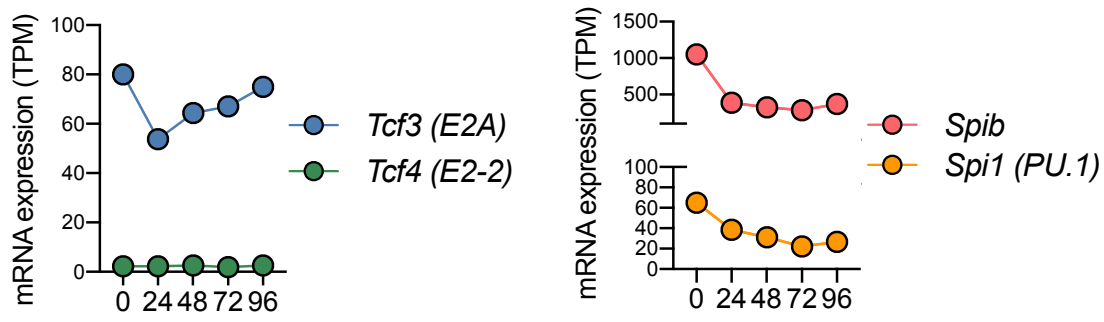

Fig. S7.

Human B lymphoblast (GM12878)

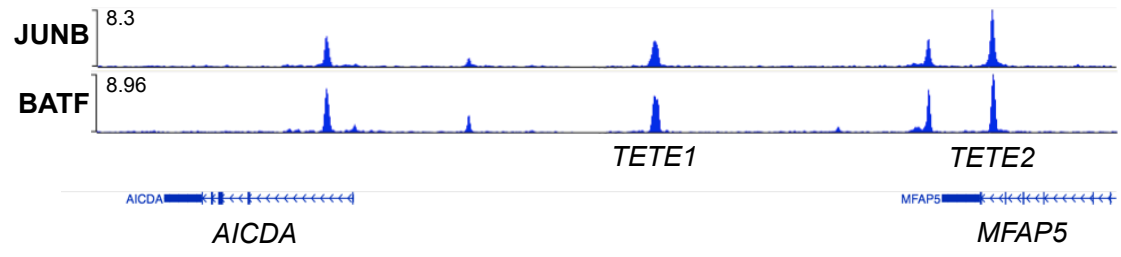

